\title{
MASS ESTIMATION THROUGH FUSION OF ASTROMETRIC AND PHOTOMETRIC DATA COLLECTION WITH APPLICATION TO HIGH AREA-TO-MASS RATIO OBJECTS
}

\author{
A Thesis \\ presented to \\ the Faculty of California Polytechnic State University, \\ San Luis Obispo
}

\author{
In Partial Fulfillment \\ of the Requirements for the Degree \\ Master of Science in Aerospace Engineering
}

by

Matthew J Richardson

June 2017 
(C) 2017

Matthew J Richardson

ALL RIGHTS RESERVED 


\section{COMMITTEE MEMBERSHIP}

TITLE:

Mass Estimation Through Fusion of Astrometric and Photometric Data Collection with Application to High Area-toMass Ratio Objects

AUTHOR: $\quad$ Matthew J Richardson

DATE SUBMITTED: June 2017

COMMITTEE CHAIR: Kira Abercromby, Ph.D.

Associate Professor of Aerospace Engineering

COMMITTEE MEMBER: Eric Mehiel, Ph.D.

Professor of Aerospace Engineering

COMMITTEE MEMBER: Morbia Jah, Ph.D.

Associate Professor of Aerospace Engineering

COMmitTeE MEMBER: Thomas Kelecy, Ph.D.

Senior Scientist, Applied Defense Solutions 


\begin{abstract}
Mass Estimation Through Fusion of Astrometric and Photometric Data Collection with Application to High Area-to-Mass Ratio Objects
\end{abstract}

\title{
Matthew J Richardson
}

This thesis work presents the formulation for a tool developed in MATLAB to determine the mass of a space object from the fusion of astrometric and photometric data. The application for such a tool is to better model the mass estimation method used for high area-to-mass ratio objects found in high altitude orbit regimes. Typically, the effect of solar radiation pressure is examined with angles observations to deduce area-to-mass ratio calculations for space objects since the area-to-mass ratio can greatly affect its orbital dynamics. On the other hand, photometric data is not sensitive to mass but is a function of the albedo-area and the rotational dynamics of the space object. Thus from these two data types it is possible to disentangle intrinsic properties using albedo-area and area-to-mass and ultimately determine the mass of a space object. Three case studies were performed for the different orbit regimes: geosynchronous, highly elliptic, and medium earth orbit. The position states were either initialized with a two line element set or with initial orbit determination methods to simulate data which was run through an unscented Kalman filter to estimate the translational and rotational states of the space object as well as the mass and albedo area. In the geosynchronous and highly elliptic cases the tool was able to accurately predict the mass value to within $\pm 5 \mathrm{~kg}$ of the true value based on a $95 \%$ confidence interval which will allow applications to understanding high area-to-mass objects with high certainty. 


\section{ACKNOWLEDGMENTS}

Thanks to:

- My committee for helping me on this endeavor

- Richard Linares for supporting me in building off his work from Reference [12] and for explaining topics I was unfamiliar with

- My parents, Gene and Christina Richardson 


\section{TABLE OF CONTENTS}

LIST OF TABLES . . . . . . . . . . . . . . . . . . . . . . . . . . . . . . . . . . . . . $\ldots$ viii
LIST OF FIGURES
CHAPTER

1. Introduction . . . . . . . . . . . . . . . . . 1

1.1 High Area-to-Mass Ratio Objects . . . . . . . . . . . . . . . 1

1.2 Mass Estimation Methods . . . . . . . . . . . . . . . . . 2

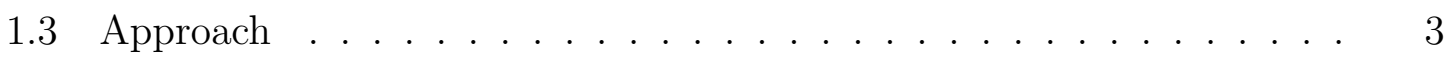

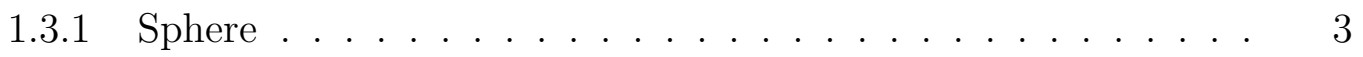

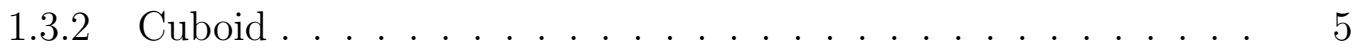

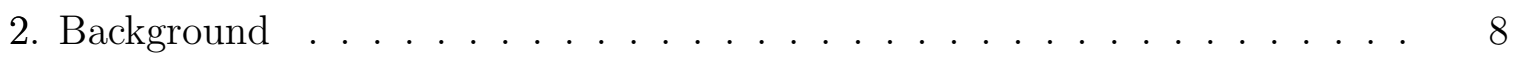

2.1 Astrometric Data . . . . . . . . . . . . . . . . 9

2.2 Photometric Data . . . . . . . . . . . . . . . . . . 9

2.3 Previous Work . . . . . . . . . . . . . . . . . 10

3. Methodology . . . . . . . . . . . . . . . . . 13

3.1 Input selection . . . . . . . . . . . . . . . 13

3.2 Shape model . . . . . . . . . . . . . . . . . . . . 14

3.3 Solar radiation model . . . . . . . . . . . . . . . . . . . . 15

3.4 Observation model . . . . . . . . . . . . . . . . . 17

3.5 Accounting for Earth Shadow . . . . . . . . . . . . . . 21

3.6 Unscented Kalman Filter . . . . . . . . . . . . . . . . . . . . 21

3.6.1 Translational \& Rotational Model . . . . . . . . . . . . 23

3.6.2 Quaternions in $\mathrm{UKF}$................. 24

3.6.3 Unscented Kalman Filter Algorithm . . . . . . . . . . . . . . 28

3.7 Mass Calculation . . . . . . . . . . . . . . . . . . . . . . . . 29

4. Case Study Design \& Analysis . . . . . . . . . . . . . . . . . . . 33

4.1 Case 1: GEO ......................... 35

$4.1 .1 \quad$ TLE . . . . . . . . . . . . . . . . . . . 35

$4.1 .2 \quad \mathrm{IOD} \ldots \ldots \ldots \ldots \ldots \ldots$ 
4.2 Case 2: HEO . . . . . . . . . . . . . . . . . . . . . . . . . 37

4.2 .1 TLE . . . . . . . . . . . . . . . . 37

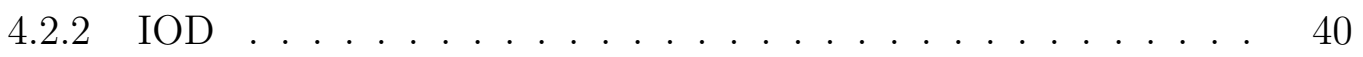

4.3 Case $3: \mathrm{MEO} \ldots \ldots \ldots$. . . . . . . . . . . . . . 40

4.3 .1 TLE .......................... 40

$4.3 .2 \mathrm{IOD} \ldots \ldots \ldots \ldots$

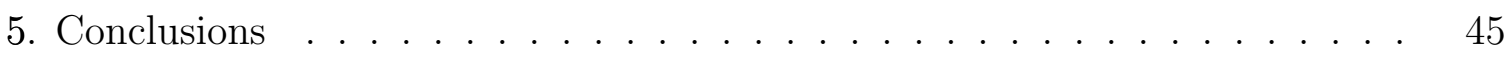

5.1 Contributions . . . . . . . . . . . . . . . . . 45

5.2 Strengths and Weaknesses . . . . . . . . . . . . . . . . 46

5.3 Future Work . . . . . . . . . . . . . . . . . . . . . . 48

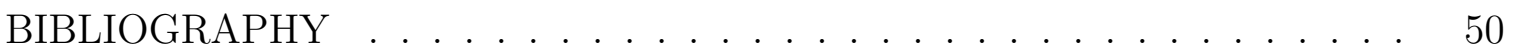
APPENDIX

A Detailed View of Result Graphs . . . . . . . . . . . . . . . 52 


\section{LIST OF TABLES}

Table

Page

3.1 UFK steps, 1 of $2 \ldots \ldots . \ldots \ldots$

3.2 UFK steps, 2 of $2 \ldots \ldots \ldots \ldots$ 


\section{LIST OF FIGURES}

Figure

Page

1.1 Albedo Area Differences Between Observer on Earth and on Sun . . 3

1.2 Sphere Position Estimate Results with $3 \sigma$ error. . . . . . . . . . . 6

3.1 Cuboid Shape Model $[12] \quad \ldots \ldots \ldots$

3.2 Observation Coordinate Frame Depiction [12] . . . . . . . . . . 18

3.3 Earths Shadow Approximation in PQR coordinates [15] . . . . . . 22

3.4 Error quaternion sequence for attitude implementation . . . . . . 26

4.1 Satellite 28884 Estimate Results . . . . . . . . . . . . . . . 36

4.2 Satellite 28868 Estimate Results . . . . . . . . . . . . . . . 38

4.3 Satellite 26464 Estimate Results . . . . . . . . . . . . . . . . . 39

4.4 Satellite 26483 Estimate Results . . . . . . . . . . . . . . . . 41

4.5 Satellite 26690 Estimate Results . . . . . . . . . . . . . . . . 42

4.6 Satellite 28874 Estimate Results . . . . . . . . . . . . . . . . 44

A.1 Satellite 28884 position estimate with $3 \sigma$ error bounds . . . . . . 53

A.2 Satellite 28884 velocity estimate with $3 \sigma$ error bounds . . . . . 53

A.3 Satellite 28884 apparent magnitude estimate with $3 \sigma$ error bounds . 54

A.4 Satellite 28884 mass estimate with $3 \sigma$ error bounds . . . . . . . . . 54

A.5 Satellite 28868 position estimate with $3 \sigma$ error bounds . . . . . . 55

A.6 Satellite 28868 velocity estimate with $3 \sigma$ error bounds . . . . . . . 55

A.7 Satellite 28868 apparent magnitude estimate with $3 \sigma$ error bounds . 56

A.8 Satellite 28868 mass estimate with $3 \sigma$ error bounds . . . . . . . . 56

A.9 Satellite 26464 position estimate with $3 \sigma$ error bounds . . . . . . 57

A.10 Satellite 26464 velocity estimate with $3 \sigma$ error bounds . . . . . . . 57

A.11 Satellite 26464 apparent magnitude estimate with $3 \sigma$ error bounds . 58

A.12 Satellite 26464 mass estimate with $3 \sigma$ error bounds . . . . . . . . 58

A.13 Satellite 26483 position estimate with $3 \sigma$ error bounds . . . . . . . 59

A.14 Satellite 26483 velocity estimate with $3 \sigma$ error bounds . . . . . . . 59 
A.15 Satellite 26483 apparent magnitude estimate with $3 \sigma$ error bounds . 60

A.16 Satellite 26483 mass estimate with $3 \sigma$ error bounds . . . . . . . . 60

A.17 Satellite 26690 position estimate with $3 \sigma$ error bounds . . . . . . 61

A.18 Satellite 26690 velocity estimate with $3 \sigma$ error bounds . . . . . . 61

A.19 Satellite 26690 apparent magnitude estimate with $3 \sigma$ error bounds . 62

A.20 Satellite 26690 mass estimate with $3 \sigma$ error bounds . . . . . . . 62

A.21 Satellite 28874 position estimate with $3 \sigma$ error bounds . . . . . 63

A.22 Satellite 28874 velocity estimate with $3 \sigma$ error bounds . . . . . . 63

A.23 Satellite 28874 apparent magnitude estimate with $3 \sigma$ error bounds . 64

A.24 Satellite 28874 mass estimate with $3 \sigma$ error bounds . . . . . . . . . 64 


\section{INTRODUCTION}

\subsection{High Area-to-Mass Ratio Objects}

Deep space optical surveys of the near geosynchronous (GEO) orbit regime have given rise to a new class of objects called HAMR or high area-to-mass ratio objects. First observed in 1999, it was hypothesized that this class of objects originated from explosions, collisions, or deterioration of resident space objects (RSO) over time [13]. HAMR objects are a particularly important set of space objects to study because their motions can pose collision hazards with GEO objects due to their large variations in eccentricity and inclination. Unfortunately, their intrinsic characteristics are unknown except for the properties for which they got their name. Their area-to-mass ratios, $\frac{C_{r} A}{m}$, can range anywhere from 0.1 to $20 \frac{m^{2}}{k g}$ [11]. HAMR objects are prone to solar radiation pressure $(\mathrm{SRP})$ on a much more significant scale than any other object in their locality. This poses a challenge for consistent cataloguing and tracking of such objects. A site might catch a passing glimpse of a HAMR object without intending to capture it, but follow up identification and association tracks prove to be non-trivial from the significant perturbation effects combined with their dim apparent timevarying reflective light intensity magnitudes. HAMR objects have difficult to predict orbit tracks that can cause potential collision hazards with the active GEO population. Thus, in order to create a reliable catalogue of the HAMR object population, it is important to accurately deduce intrinsic characteristics such as area, albedo, and mass. 


\subsection{Mass Estimation Methods}

Commonly, mass is determined indirectly using the effective albedo area-to-mass ratio that comes out of current orbit determination processes. This value is based on solar radiation pressure. That value is then divided by albedo area that is based on photometry. Due to dynamic mismodeling of space objects this can sometimes lead to inconsistent predictive results. The issue for mass estimation methods is to accurately fuse astrometric data and photometric data together to extract where each has its strengths.

The angles measurements are very sensitive to the effective albedo area-to-mass ratio when it comes to solar radiation pressure while the photometry is sensitive to albedo area products where the albedo area is not solar pressure albedo but instead the observed albedo. This observed albedo area will always be a fraction of the solar pressure albedo area, but they are not the same. These two quantities are only the same at zero phase angle or if the observer is standing on the sun. An example of this is illustrated in Figure 1.1. It is evident that the area which is facing an observer will be different than the side exposed to the incident flux of the Sun. Thus, due to the material properties and the exposed area of the space object, the albedo value from the optical site will measure one thing which would be different from if the observer were to measure the albedo of the space object from the point of view of being on the Sun.

This topic has importance to the entire orbital space community due to the unpredictable nature of HAMR objects. Methods of initial orbit determination need a survey of multiple nights worth of consistent data to stitch together a hypothesized orbit track for a certain space object. Due to the significant effect of solar radiation pressure on these HAMR object's eccentricities and inclinations, they can be within a field of view one night and thrown into a completely different orbit by the next ob- 


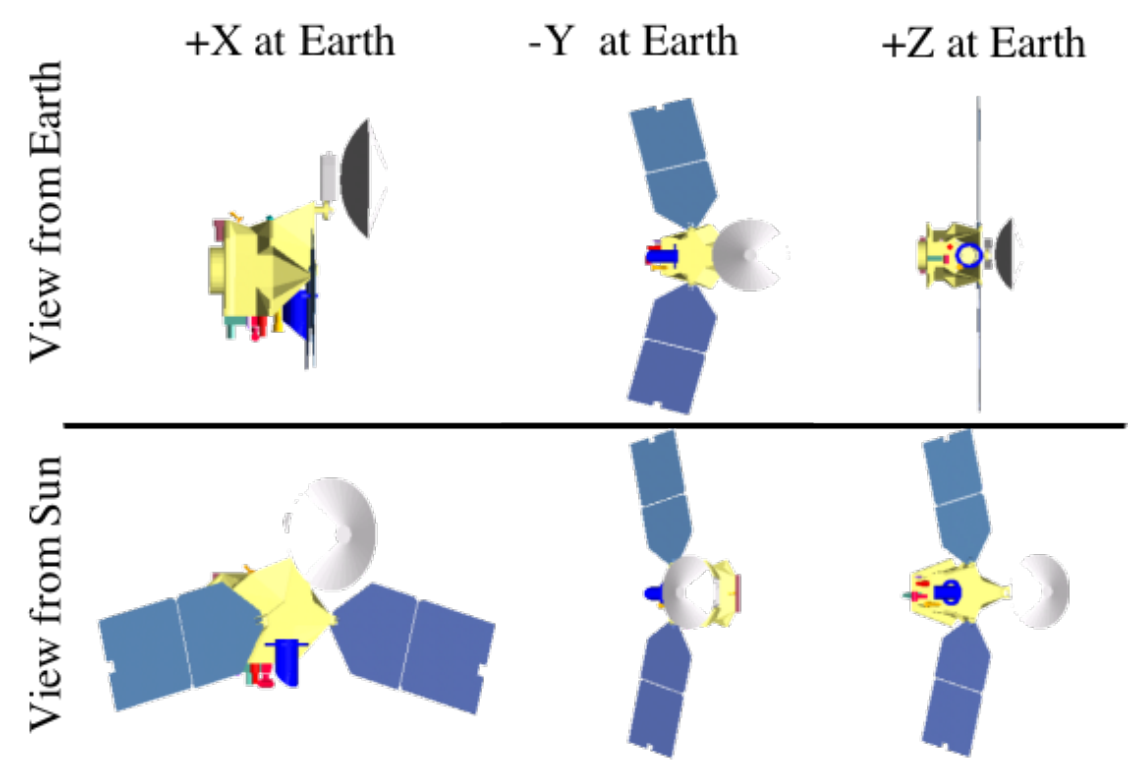

Figure 1.1: Albedo Area Differences Between Observer on Earth and on Sun

serving time frame. Thus it is important to identify and catalog these objects within as short of a period of time as possible as to not lose sight of them. Once that feat is accomplished, keeping track of a particular HAMR object and studying its characteristics will allow further information to be gained about this class of space debris. Additionally, studying these objects more closely will provide further information into the origin and dynamics of other similar space objects.

\subsection{Approach}

\subsubsection{Sphere}

The approach to determining mass of an unknown space object requires numerous assumptions and simplifications about the unknown nature of the observed object. To begin this journey a simple spherical model of an object was used to test the set up the proper passing of information through the unscented Kalman filter (UKF). Details 
of the UKF will be detailed in Section 3.6. Solar radiation pressure was modeled by

$$
\mathbf{a}_{s r p}^{I}=\frac{-p_{s r p}(1+a) A}{m} \mathbf{u}_{s u n}
$$

where $p_{s r p}$ is the pressure due to solar radiation, $a$ is the albedo of the uniform sphere, $A$ is the area, $m$ is the mass, and $\mathbf{u}_{\text {sun }}$ is the unit vector pointing from the object towards the Sun.

The photometric flux was measured by

$$
F=\Phi_{\text {sun }} \frac{R_{\text {diff }} A}{r^{2}}\left(1+\mathbf{u}_{\text {obs }} \cdot \mathbf{u}_{\text {sun }}\right)
$$

where $\Phi_{\text {sun }}$ is the average incident radiant flux density from the Sun at $1 \mathrm{AU}$, given by $\Phi_{\text {sun }}=1,367 \mathrm{~W} / \mathrm{m}^{2}, R_{\text {diff }}$ is the diffuse coefficient of reflectively which is just equal to the albedo for this calculation, $\mathbf{u}_{o b s}$ is unit direction the observer is viewing the space object from, and $r=\|\mathbf{r}\|$.

It follows that the equations of motion were then represented by

$$
\mathbf{f}(\mathbf{x})=\left[\begin{array}{c}
\dot{\mathbf{r}}^{I}=\mathbf{v}^{I} \\
\ddot{\mathbf{r}}^{I}=-\frac{\mu}{r^{3}} \mathbf{r}^{I}+\mathbf{a}_{s r p}^{I} \\
\dot{A}=0 \\
\dot{m}=0
\end{array}\right]
$$

where $\mu$ is the gravitational parameter of Earth and $\mathbf{a}_{s r p}^{I}$ represents the acceleration perturbation due to SRP in the inertial frame. The state vector $\mathbf{x}$ carried with it the position, velocity, area, and mass of the sphere. For this case, $\dot{m}=0$ and $\dot{A}=0$. While these simplifications were not able to produce significant results, the development of this mass estimation tool started with understanding how to properly code a UKF. Thus, they were used as a double check that the UKF was properly running and providing results where the $3 \sigma$ error bounds were converging within an appropriate time frame and the measurements were staying well within those bounds. 
The details of how the UKF was coded can be found in Section 3.6 but the approach was to take an a priori estimate for time step $t_{k-1}$, pass that through the equations of motion for astrometric information, use the updated a posteriori information and a measurement value from the photometric information to produce an updated measurement of the state at time $t_{k}$, update that with a Kalman gain, and proceed to the next time step using that new state vector as the a priori data value. Figure 1.2 is an example output from the UKF and how the rest of the work will determine the validity of the model. The red dots in this figure are the difference in estimated values from the UKF output state and what is considered to be the 'truth' state. If the UKF was accurate and working properly we should see a convergence to the value 0 as the UKF would be picking up the true value. Again, more details will be discussed in Section 3.6 but this is the general overview and was an important step in the approach because the UKF needed to be working before any more state information could be added and more complex equations of motion could be propagated.

\subsubsection{Cuboid}

Once certain the UKF was working properly, Equations (1.1) and (1.2) would be extended to a more robust shape model that would be more indicative of a true spacecraft as well as one that could be fed attitude and spin information to discern one face from another. For these purposes, a cuboid model was chosen to evolve from the spherical model for the continuation of experimentation. The cuboid was defined by three parameters: $d, w, h$, which are the depth, width, and height, respectively. An example of this cube can be seen in Figure 3.1 and the necessary equations for calculation will be further discussed in Section 3.2.

This approach was much more realistic in comparison to Section 1.3.1 since a 

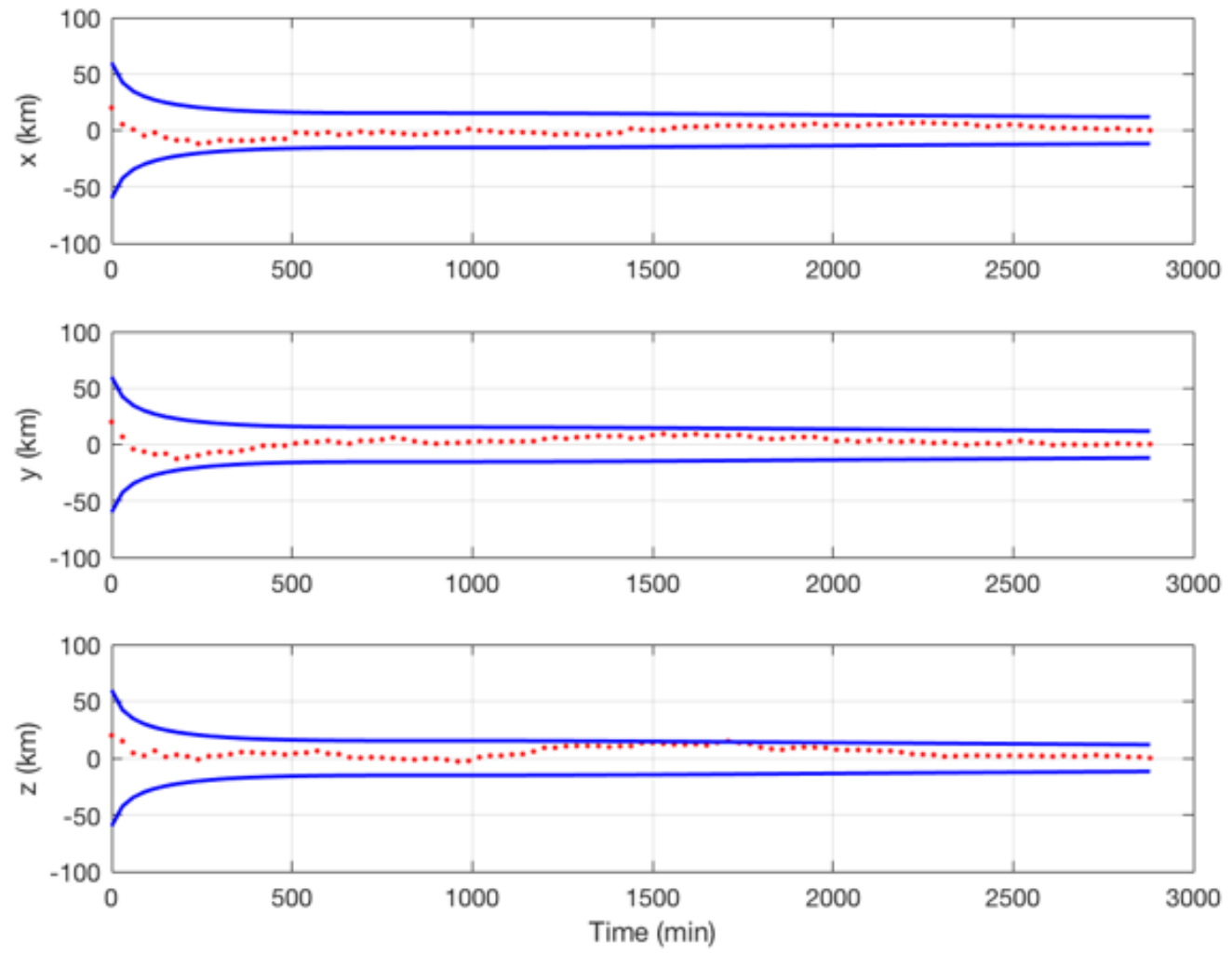

Figure 1.2: Sphere Position Estimate Results with $3 \sigma$ error. 
perfect homogeneous sphere is not a good approximation for most space object. The simplifications of a spherical model do not provide enough information to accurately keep track of the important intrinsic properties that would allow for proper mass estimation and orbit determination. Having a finite number of facets each with their own albedo, area, and orientation allows the algorithm to be more robust for future applications. The details of the more robust equations of motion and observation will be outlined in Chapter 3. 


\section{BACKGROUND}

With astrometric, or angles data, a sensor will take pictures of the night sky over a certain period of time. A reduction process is conducted to determine the location of the streak in the night sky through comparison of known stars and thereby computing the angular information. For photometric data, or light information coming from a charge-coupled-device camera (CCD), the magnitude and direction of a particular light source will give information on the space object. The challenge when applying this to HAMR objects as discussed in Section 1.1 is that astrometric data gives you positional information and, hence, perturbations on the orbital dynamics that are indirectly related to the photometric signature. The photometric signatures gives you attitude related information which effects the positional perturbations. An investigation into coupling the strengths of each data type to determine characteristics such as mass is the primary focus of this work.

This work will use an unscented Kalman filter as a means for estimation, but a method for initializing the track was also needed. One approach utilized in many orbit determination applications is to perform an Initial Orbit Determination (IOD) step and then refine the initial estimate provided by IOD via a Batch Processor mechanism (e.g. a Square-Root Information Filter) before transitioning to sequential estimation with a mechanism such as the UKF [5]. Refining the initial estimate is outside the scope of this work. The technique used here for IOD is the extended Gauss iteration $[7],[10]$.

Gauss iteration is an angles only solution to initial orbit determination that uses three different observations as a method to formulate a position and velocity vector for a space object. The formulation derives a line of sight vector by the right ascension 
and declination values, a site vector described using the local sidereal time of each observation, and the time difference between each observation. Gauss' method was chosen in comparison to the other IOD methods, such as double $\mathrm{r}$ or Izzo-Gooding [15], because it is better at handling objects which do not have a lot of angular separation. This made it ideal for the purposes of this work due to the short data collection time window of HAMR objects in orbit and the GEO regime which the objects reside in. However it is important to be aware of the limitations of Gauss' method such as its sensitivity to data noise and inefficiency with collections spanning more than $60^{\circ}[15]$.

\subsection{Astrometric Data}

It has been shown in [11] that angles data can produce information about a areato-mass ratio due to SRP forces. However, that study was restricted in terms of rotational dynamic modeling. When combined with the typically dim magnitudes of HAMR objects, the result was lost or incorrectly associated space objects. To accurately describe the translational and rotational dynamics of a known object data must be consistently taken over a span of multiple days for that same object. Unfortunately, this is not feasible for something like a HAMR object since the drastic changes in eccentricity and inclination can kick the object out of a known orbit track within a period of a few hours. With the attempt of infusing the UKF with periodic photometric measurements, mass properties should be more accurately modeled.

\subsection{Photometric Data}

Light curves are able to estimate the shape and albedo properties of a space object. In particular, Reference [9] was able to deduce the rotation period, pole direction, and scattering parameters of an asteroid using only light curve information. Since magni- 
tude information can also be used to deduce information about position and attitude and their respective rates [12] utilizing photometric information can be considerably useful in determining mass information for a space object. This is because there are multiple ways to approach the modeling information of intrinsic parameters which are all not sensitive to the same restrictions. Thus, when incorporated in conjunction, astrometric and photometric data can each provide useful characteristics to deduce mass.

\subsection{Previous Work}

This work was built upon the previously developed application from Linares et. al in Reference [12]. Dr. Linares was instrumentally helpful in explanations for the process of unscented Kalman filtering which will be described in Section 3.6 as well as in code exchanges for the application of quaternions in the UKF seen in Section 3.6.2. Since the code was not produced for public use, this code in this work was built from the ground up to mimic the types of results obtained in [12] with the intention of allowing others to build upon this research in the future in an attempt to derive a better public tool for mass estimation.

In Reference [12], Linares et. al approach the topic of astrometric and photometric data fusion via three different methods, each with their own unique parameters that are estimated for in the state vector and then carried through the UKF. In the first method, they estimate the mass using an assumed value for the albedo. The second method makes use of the fact that the observations and dynamics are both sensitive to the desired mass and area quantities but by themselves are not sensitive to all the estimated state values. And finally method three does not assume albedo values and estimates for the albedo directly.

The work conducted in [12] framed the structure which the work in this thesis 
followed. Both used a unscented Kalman filtering estimation scheme using light curve and angles data to update the measurement information for the state and covariance matrices at different collection intervals. Using the same cuboid shape object and similar parameters, Linares et. al were able to converge on a mass estimation for their space object of mass $1,500 \mathrm{~kg}$ to within $54.76 \mathrm{~kg} 3 \sigma$. The maximum area-tomass ratio was 0.09 for that cuboid object. When run with different area-to-mass ratios of $0.01,0.1,1$, and 10 the mass estimation converged to $3 \sigma$ errors of $160.12 \mathrm{~kg}$, $30.45 \mathrm{~kg}, 2.71 \mathrm{~kg}$, and $0.80 \mathrm{~kg}$ respectively. These results were incredibly accurate for the objects in question and the multiple approach method provided opportunities to apply mass estimation in different ways depending on what sort of information you have available and what you would like to estimate.

Reference [2] adopts a similar formulation for finding the area and mass information of a space object combining astrometric and photometric data but modeled as a sphere. In this study a sphere was a good enough approximation for the model because it was meant to compare the relative estimation abilities of two sensor platforms, not evaluate absolute estimation accuracy in a real world setting. However, the applications between the two are very similar since the paper showed an advantage of reducing the targets mass uncertainty very quickly with both astrometric and photometric data.

Reference [8] describes the formulation for a filter framework Optical Data Estimation for Space Situational Awareness (ODESSA) which works to fuse astrometric and photometric data in near real time for state estimation. ODESSA also has a A backward smoother capability as a means of recovering the best possible estimates based upon all of the data available. Jah and Madler were able to show promising convergence of errors when using an unscented Kalman filter formulation, much like this work employs. 
The research in this paper expands upon each of these ideas and examines the strengths of implementing these techniques in different orbit regimes. The ultimate goal is to apply this modeling to HAMR objects which reside in the GEO regime thus that is the main focus during development. 


\section{METHODOLOGY}

The ultimate goal of this work is to develop a tool in MATLAB capable of mass estimation for space objects in different orbit regimes as well as the ability to apply the gained knowledge and understanding to HAMR object research. This was accomplished by understanding how to properly combine and propagate two different types of information, angles data and light curve data, through an unscented Kalman filter. This method of filtering was chosen mainly because it allowed a means of estimating information about the states using a priori and a posteriori information. Thus as the space object was propagating, measurements of light curve data could be used to update the state information with less error over time, thus converging as more measurements are taken.

\subsection{Input selection}

There are two built-in methods within the tool for obtaining a position and velocity vector to describe the orbit of the space object. The first is for a well catalogued space object such as a satellite with a registered NORAD Catalog ID number. By

providing the input file with the catalog ID number and a Julian date of interest, the code will pull the closest TLE to the provided Julian date number. Utilizing that TLE, you can specify a desired Julian date that the position and velocity vectors will be propagated to utilizing Vallado's SGP4 propagator [14]. From there the vector states will be fed into the UKF for processing.

The second method incorporates initial orbit determination (IOD) method discussed in Section 2 in order to initialize the position and velocity vectors of the space 
object. Utilizing a set of three angles observations, with right ascension and declination values, position and velocity vectors can be found through a Gauss iteration. This formulation is more efficient when the angular separation between observations is less than about $60^{\circ}$ and will perform remarkably well for data separated by $10^{\circ}$ or less. This method was chosen for the tool because in particular for HAMR object there might not be a large window of time you have to collect on the object because of the large changes in orbital parameters due to SRP. Thus, with only a few small collections, there needed to be a way for the tool to handle determining the initial orbit of the space object.

Both methods have consistent information that will be used by either method, including the information about the optical site such as longitude, latitude, and height above sea level. Additionally, input information about time of the observations and length of time the UKF should propagate from the initialization must be included for the program to carry out the proper iterations.

\section{$3.2 \quad$ Shape model}

The cuboid shape utilized in this experimentation was previous defined in Section 1.3.2 and was illustrated in Figure 3.1. The finite number of flat facets, six in the case of the cuboid, allowed for information to be propagated through the experimentation as the object was allowed to rotate. Each facet had a set of three basis vectors to parametrize the surface $\left\langle\mathbf{u}_{u}^{B}, \mathbf{u}_{v}^{B}, \mathbf{u}_{n}^{B}\right\rangle$ where $\mathbf{u}_{u}^{B}$ and $\mathbf{u}_{v}^{B}$ were in the plane of the facet and $\mathbf{u}_{n}^{B}$ was normal to the facet. Because the space object was considered to be a rigid body the unit basis vectors did not change with time being expressed in the body frame of reference.

The SRP and light curve models require these vectors to be expressed in the inertial coordinate system. To do so and account for the rotational dynamics of the 


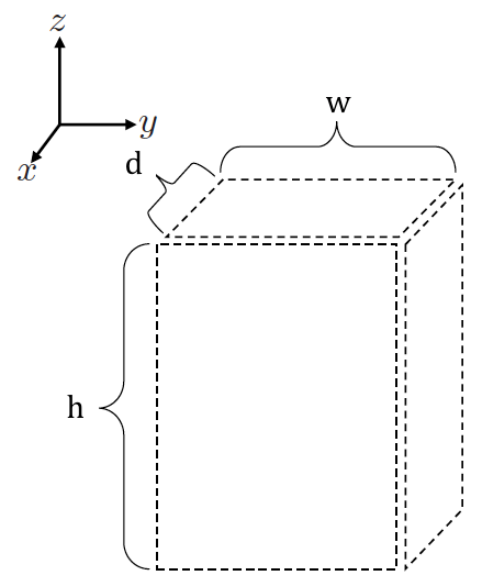

Figure 3.1: Cuboid Shape Model [12]

space object we can map the body reference frame to the inertial reference frame using an attitude matrix $A\left(\varrho_{I}^{B}\right)$ given by

$$
\mathbf{u}_{k}^{B}=A\left(\varrho_{I}^{B}\right) \mathbf{u}_{k}^{I}, \quad k=u, v, n
$$

where $\varrho_{I}^{B}$ is the quaternion of the state. The six sides of the cuboid space object each had a surface facet unit normal to keep track of when the object was rotating in the inertial frame. The area vector $A$ has dimension $n$ by 1 where $n$ is the number of facets the object has. This vector was propagated alongside the equations of motion but should remain unchanged as the object orbits on account of the assumption of a rigid body.

\subsection{Solar radiation model}

For objects orbiting at high altitudes $(\geq 1,000 \mathrm{~km})$ the dominating non conservative perturbation force is solar radiation pressure (SRP). To accurately describe the effects SRP has on a space object, position and attitude dynamics must be coupled together. Additionally, parameters such as reflectivity and the solar flux arriving at the space 
object's position (accounting for shadowing effects) must be correctly determined. This work assumes a constant average flux coming from the Sun at all periods where in all reality this simplification would not be true for periods of intense solar activity, when the value would be much larger or conversely in periods of low activity when the effect might be negligible. Because the incoming radiation from the Sun exerts a force on the space object, the apparent size of the space object that faces the Sun is critical in accurately determining the amount of acceleration. According to [15] the force can be modeled as

$$
\mathbf{F}_{s r p}=-p_{s r p} c_{R} A \odot \frac{\mathbf{r}_{s o \odot}}{r_{s o \odot}}
$$

where $p_{s r p}$ is the pressure due to solar radiation, $c_{R}$ is the coefficient of reflectivity, $A_{\odot}$ is the exposed area to the Sun, and $r_{s o \odot}$ is the position vector from the space object to the Sun. In this case the average solar pressure is modeled by

$$
p_{\text {srp }}=\frac{\Phi_{\text {sun }}}{c}=\frac{1467}{3 \times 10^{8}} \frac{\mathrm{W} / \mathrm{m}^{2}}{\mathrm{~m} / \mathrm{s}}=4.57 \times 10^{-} 6 \frac{\mathrm{N}}{\mathrm{m}^{2}}
$$

acceleration is obtained by using Newton's second law with the given force

$$
a_{s r p}=\frac{F_{s r p}}{m}=\frac{-p_{s r p} c_{R} A_{\odot}}{m}
$$

Equation (3.4) is a jumping off point to obtaining the proper model used in this work. Equation (1.1) was a simplified model for a sphere without considering the differences in reflectivity. Due to the material property of a space object, some sides will reflect diffusely while others will reflect specularly. Hence, at any moment the space object will experience a net force plus a net torque. Considering this, the angle at which the solar radiation is incident on a surface element of area $A_{\odot}$, whose surface normal vector makes a solar-incident angle $\phi_{\text {inc }}$ with the Sun-space object line. In assuming the diffuse and specular radiation forces are Lambertian diffusion, [6] derives the acceleration due to solar radiation pressure acting on a body composed 
of $n$ flat plates as

$$
\mathbf{a}_{s r p}^{i}=-p_{s r p}\left(\frac{r_{a u}}{r_{s o}}\right)^{2} \sum_{k=1}^{n} \frac{A_{k}}{m} \cos \phi_{k}\left[\left(1-\rho_{k}\right) \mathbf{u}_{s u n}^{I}+2\left(\frac{1}{3} \delta_{k}+\cos \phi_{k} \rho_{k}\right) \mathbf{u}_{n, k}^{I}\right],
$$

where $r_{a u}$ is the distance of one astronomical unit, $r_{s o}=\left\|\mathbf{r}_{\text {sun }}^{I}-\mathbf{r}^{I}\right\|$ is the distance of the Sun with respect to the space object, $m$ is the total space object mass, $A_{k}$ is the area of the $k^{t h}$ plate, $\rho_{k}$ is the specular reflection coefficient of the $k^{\text {th }}$ plate, $\delta_{k}$ is the diffuse reflection coefficient of the $k^{\text {th }}$ plate, $\mathbf{u}_{n, k}^{I}$ is the unit vector normal to the $k^{\text {th }}$ plate expressed in the inertial frame using Equation (3.1). The solar-incident angle $\phi_{k}$ is given by

$$
\cos \phi_{k}=\mathbf{u}_{n, k}^{I} \cdot \mathbf{u}_{\text {sun }}^{I}
$$

where $\mathbf{u}_{\text {sun }}^{I}$ is the unit vector space object to the Sun expressed in the inertial reference frame, which is given by

$$
\mathbf{u}_{\text {sun }}^{I}=\frac{\mathbf{r}_{\text {sun }}^{I}-\mathbf{r}^{I}}{\left\|\mathbf{r}_{\text {sun }}^{I}-\mathbf{r}^{I}\right\|}
$$

\subsection{Observation model}

Observations for flux and magnitude measurements were made considering an optical site located at some latitude $\lambda$, local sidereal time $\theta$, and East Longitude $\phi$ from the observed space object. The geometry associated with the space object with respect to the optical site can be seen in Figure 3.2 where $\mathbf{d}^{I}$ is the position vector from the observer to the space object, $\mathbf{r}^{I}$ is the position vector of the space object in EarthCentered Inertial (ECI) coordinates, and $\mathbf{R}^{I}$ is the geocentric position vector locating the optical site. Thus the position vector $\mathbf{d}^{I}$ can be calculated as

$$
\mathbf{d}^{I}=\mathbf{r}^{I}-\mathbf{R}^{I}
$$




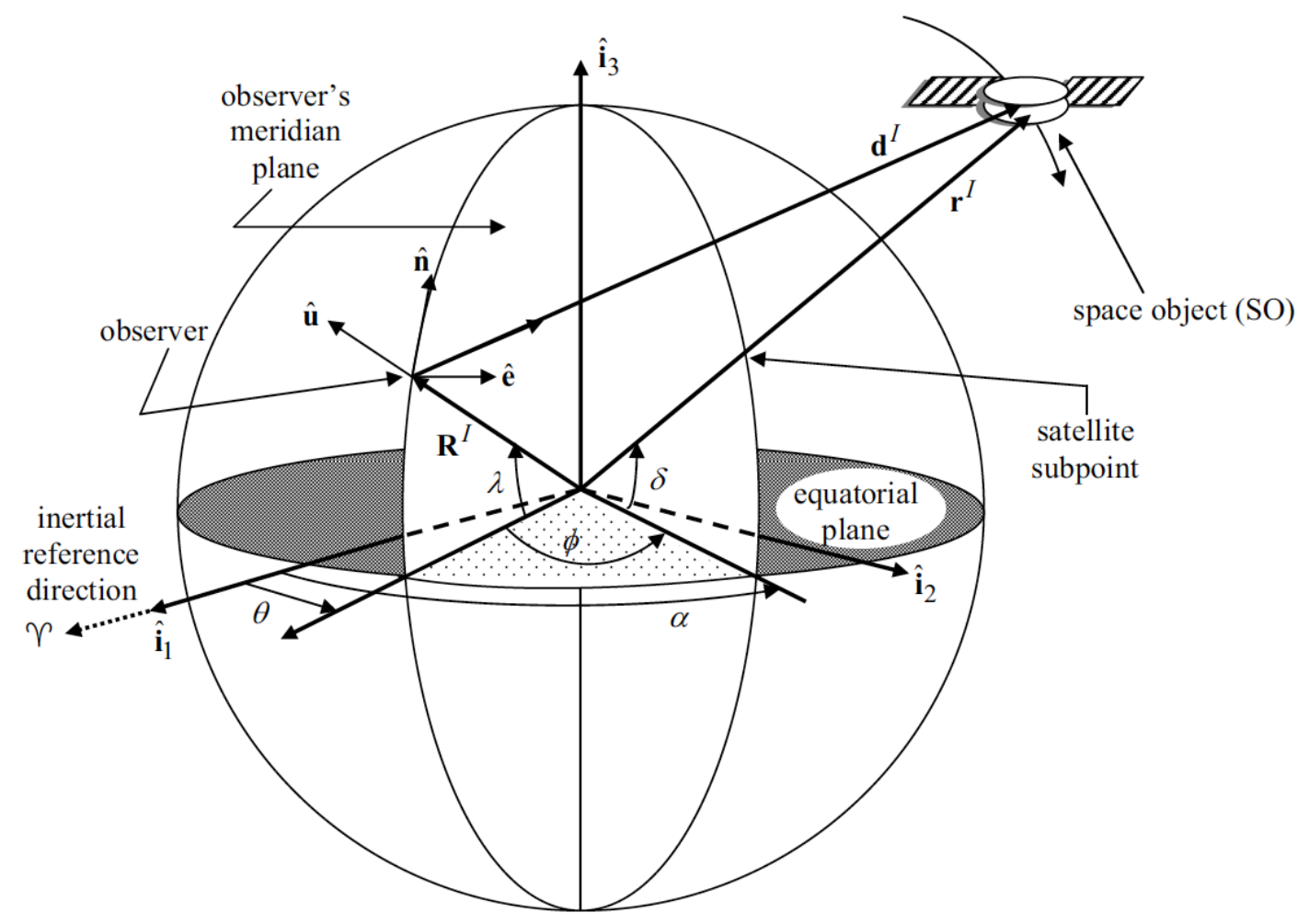

Figure 3.2: Observation Coordinate Frame Depiction [12] 
The geocentric position vector, $\mathbf{R}^{I}$, of the optical site is calculated using [4],

$$
\begin{gathered}
\mathbf{R}^{I=}\left[\frac{R_{\oplus}}{\sqrt{1-\left(2 f-f^{2}\right) \sin ^{2} \phi}}+H\right] \cos \phi(\cos \theta \hat{\mathbf{i}}+\sin \theta \hat{\mathbf{j}}) \\
+\left[\frac{R_{\oplus}(1-f)^{2}}{\sqrt{1-\left(2 f-f^{2}\right) \sin ^{2} \phi}}+H\right] \sin \phi \hat{\mathbf{k}}
\end{gathered}
$$

Where $R_{\oplus}$ is the radius of the Earth, $f=0.003353$ and is the oblateness factor of the Earth, $\mathrm{H}$ is the elevation, $\phi$ is the geodetic latitude, and $\theta$ is the east longitude of the site. A graphic representation of these values can be seen in Figure 3.2.

Lastly, the position vector $\mathbf{d}^{I}$ must be transformed into the topocentric horizon coordinate system, often referred to as the Up-East-North coordinate system. The conversion is given by

$$
\boldsymbol{\rho}^{I}=\left[\begin{array}{ccc}
-\sin \theta & \cos \theta & 0 \\
-\sin \phi \cos \theta & -\sin \phi \sin \theta & \cos \phi \\
\cos \phi \cos \theta & \cos \phi \sin \theta & \sin \phi
\end{array}\right] \mathbf{d}^{I}
$$

The topocentric horizon position vector $\boldsymbol{\rho}^{I}$ is then converted into a unit vector for further calculation use, such as in Section 3.5 for the vector calculation for the Earth's shadow and as will be seen for the specular and diffuse reflection calculations.

The optical site records position of the space object as well as the magnitude of the brightness. That brightness can be modeled using a bidirectional reflectance distribution function (BRDF) which is a way to model light scattered from the surface of an object due to incident light. The BRDF at any point on the surface is a function of two directions: the direction from which the light source originates and the direction from which the scatter light leave the surface [12]. Thus the total BRDF is a sum of a specular and a diffuse component given by

$$
\rho_{\text {total }}(i)=\rho_{\text {spec }}(i)+\rho_{\text {diff }}(i)
$$

The diffuse component is Lambertian diffusion, scattering the light equally in all directions while the specular component are the mirror-like characteristics, concentrating 
the light in one particular location. Reference [1] develops a BRDF for modeling continuous arbitrary surfaces but simplifies it for flat surfaces. This can then be applied to the $n$ sided flat surface model employed in this work. Under this flat plate simplification [1]

$$
\rho_{\text {spec }}(i)=C_{\text {spec }} \frac{\left(\mathbf{u}_{\text {obs }} \cdot \mathbf{u}_{\text {spec }}\right)^{\alpha}}{\mathbf{u}_{\text {sun }} \cdot \mathbf{u}_{n}},
$$

where $\mathbf{u}_{\text {spec }}=2\left(\mathbf{u}_{n} \cdot \mathbf{u}_{\text {sun }}\right) \mathbf{u}_{n}-\mathbf{u}_{\text {sun }}$ and is the unit vector in the direction of specular reflection, $C_{\text {spec }}$ is the coefficient of specular reflectivity, and $\alpha$ defines the width of the specular lobe, $\mathbf{u}_{n}^{I}(i)$ is the unit normal of the $i^{\text {th }}$ plate, and $\mathbf{u}_{\text {sun }}$ is the unit vector of the Sun's direction. Then the diffuse component is given by

$$
\rho_{\text {diff }}(i)=\frac{C_{d i f f}}{\pi}
$$

where $C_{\text {diff }}$ is the coefficient of diffuse reflectivity. Knowing this the flux equations can be developed. The flux of visible light which reaches the surface of the space object is modeled by

$$
F_{\text {sun }}(i)=\Phi_{\text {sun }, \text { vis }} \rho_{\text {total }}(i)\left(\mathbf{u}_{n}^{I}(i) \cdot \mathbf{u}_{\text {sun }}^{I}\right)
$$

where $\Phi_{\text {sun }, \text { vis }}=455 \mathrm{~W} / \mathrm{m}^{2}$ is the average power per square meter from the sun striking the surface of the object and the unit vectors are as previously defined. Keeping in mind that if for any side the angle between the surface normal and the Sun's direction was larger than $\pi / 2$, than the solar flux would be set to zero since there was no radiant flux on that facet. Then the reflected light becomes

$$
F_{\text {obs }}(i)=\frac{F_{\text {sun }}(i) A(i)\left(\mathbf{u}_{n}^{I}(i) \cdot \mathbf{u}_{\text {sun }}^{I}\right)}{\left\|\mathbf{d}^{I}\right\|^{2}}
$$

where $A(i)$ is the area of the $i^{\text {th }}$ facet and the other terms are as previously defined. Finally, the apparent magnitude can be calculated as

$$
m_{\text {app }}=-26.7-2.5 \log _{10}\left|\sum_{i=1}^{n} \frac{F_{\text {obs }}(i)}{\Phi_{\text {sun }, v i s}}\right|
$$

where -26.7 is the apparent magnitude of the Sun. 


\subsection{Accounting for Earth Shadow}

The solar radiation model and observation model were equipped with the ability to handle shadow calculations for when a spacecraft is passing in the shadow of the Earth such as can be seen in Figure 3.3. To do so, the translational and rotational model considers the current position vector of the space object and the current position vector of the Sun. The angle between the two objects with the common central body of Earth can be found using

$$
\theta=\cos ^{-1}\left(\frac{\mathbf{r}_{\odot} \cdot \mathbf{r}_{s o}}{r_{\odot} r_{s o}}\right)
$$

where $\mathbf{r}_{\odot}$ is the position vector of the Sun, $\mathbf{r}_{s o}$ is the position vector of the space object, $r_{\odot}=\left\|\mathbf{r}_{\odot}\right\|$, and $r_{s o}=\left\|\mathbf{r}_{s o}\right\|$. Then each object makes an tangent line with the edge of Earth, which forms a triangle with vertex angle given by

$$
\theta_{s o}=\cos ^{-1}\left(\frac{R_{\oplus}}{r_{s o}}\right) \quad \theta_{\odot}=\cos ^{-1}\left(\frac{R_{\oplus}}{r_{\odot}}\right)
$$

where $R_{\oplus}$ is the radius of the Earth, meaning that there is no line of site between

the space object and the sun when $\theta_{s o}+\theta_{\odot}<\theta[4]$. Each unit normal vector of the facet was constantly checked with the vector position of the sun. If the addition of the two angles were greater than the sum of the angle between them then SRP and measurement fluxes were set to zero.

Reference [11] shows that using a simple cylindrical Earth shadow model can result in errors in the force modeling. These errors should be accounted for in future work if attempting to obtain the highest accuracy for the orbit kinematics.

\subsection{Unscented Kalman Filter}

Filtering algorithms are commonly used for state estimation of space objects. This allows for estimations of unknown variables through the calculation of a state prob- 


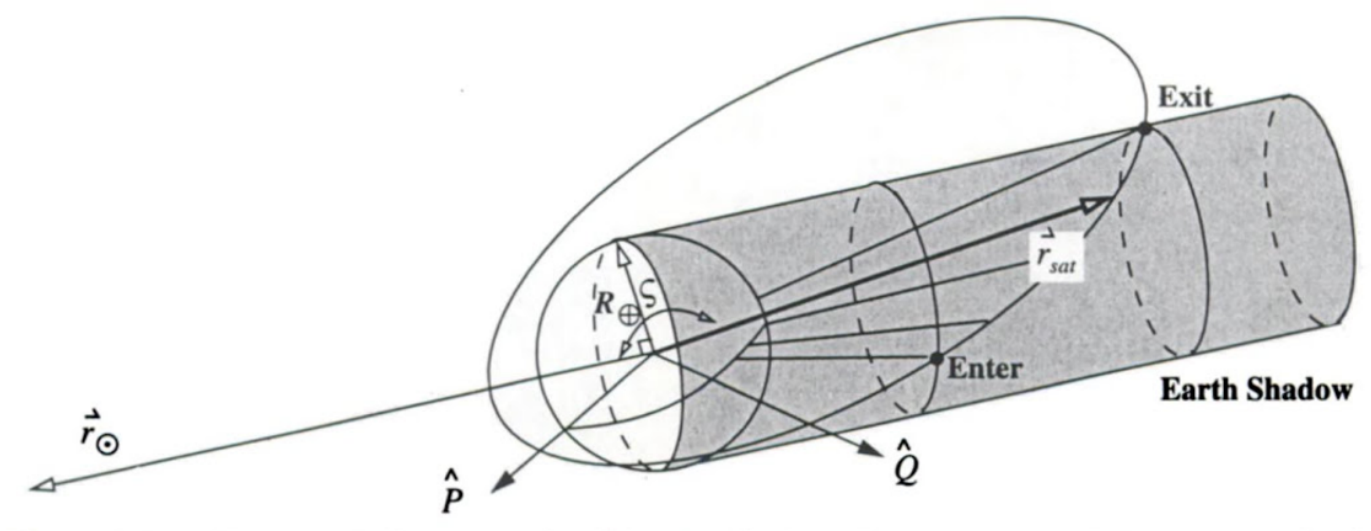

Figure 3.3: Earth's Shadow Approximation in PQR coordinates [15]

ability density function (pdf) using a posteriori or a priori information. In other words when information is known about the past or future states of the space object, the UKF can model the unknown parameters using weighted sigma points which are state vectors deterministically sampled about a mean. These are used to parametrize the true mean and covariance of a state distribution. Once propagated through a nonlinear system the posterior mean and covariances are obtained and updated through a weighting process. This update process changes based on the number of measurements gained between each time step and thus make adjustments to the state mean and covariance as it propagates.

The mean and covariance variables are determined via a distribution of sigma points obtained from the filter estimations. Given an $L \times L$ error covariance matrix $P_{k}$, the sigma points are constructed by [12]

$$
\begin{gathered}
\boldsymbol{\sigma}_{k} \equiv \pm \sqrt{(L+\gamma) P_{k}} \\
\chi_{k}(0)=\boldsymbol{\mu}_{k} \\
\chi_{k}(i)=\boldsymbol{\mu}_{k}+\boldsymbol{\sigma}_{k}(i)
\end{gathered}
$$

Given that these points are selected to represent the distribution of the state vector, 
each sigma point is weighted to preserve the initial condition information

$$
\begin{gathered}
W_{0}^{\text {mean }}=\frac{\lambda}{L+\lambda} \\
W_{0}^{c o v}=\frac{\lambda}{L+\lambda}+\left(1-\alpha^{2}+\beta\right) \\
W_{i}^{\text {mean }}=W_{i}^{\text {cov }}=\frac{1}{2(L+\lambda)}, \quad i=1,2, \ldots, 2(L-1)
\end{gathered}
$$

where $\lambda=\alpha^{2}(L+\kappa)-L$ is a scaling parameter, $\alpha$ is a parameter that controls the spread of sigma points and should be $0<\alpha \leq 1, \kappa=3-L$, and $\beta$ is used to incorporate prior knowledge of the distribution by weighing the mean sigma points in the covariance matrix [12].

Applying the UKF did not come without challenges however. As will be discussed in Section 3.6.2, quaternions are not as easily propagated through the filter like other state elements.

\subsubsection{Translational \& Rotational Model}

The dynamic modeling which the UKF utilizes its unscented transform on is a nonlinear rotational and translational model parametrized by

$$
\mathbf{f}([\boldsymbol{\chi}, \hat{\boldsymbol{\varrho}}])=\left[\begin{array}{c}
\hat{\mathbf{v}}^{I} \\
-\frac{\mu}{r^{3}} \hat{\mathbf{r}}^{I}+\hat{\mathbf{a}}_{s r p}^{I} \\
\frac{1}{2} \Xi(\hat{\boldsymbol{\varrho}}) \hat{\boldsymbol{\omega}}_{B / I}^{B} \\
J_{S O}^{-1}\left(\hat{\mathbf{T}}_{s r p}^{B}-\left[\hat{\boldsymbol{\omega}}_{B / I}^{B} \times\right] J_{S O} \hat{\boldsymbol{\omega}}_{B / I}^{B}\right)
\end{array}\right]
$$

where the first component is velocity, the second component is the acceleration due to solar radiation pressure, the third component is the quaternion derivative and the last component is the angular momentum with an included torque. The superscripts $I$ account for the inertial reference frame and $B$ account for the body frame of the space object. The acceleration component due to solar radiation pressure was calculated 
using Equation (3.5). The angular momentum component is comprised of the inertia matrix, $J_{S O}$, and the net torque due to solar radiation pressure, $\mathbf{T}_{s r p}^{B}$. For the cuboid shape discussed in Section 3.2, the inertia matrix is

$$
J_{S O}=m\left[\begin{array}{c}
\frac{d^{2}+h^{2}}{12} \\
\frac{w^{2}+d^{2}}{12} \\
\frac{h^{2}+w^{2}}{12}
\end{array}\right]
$$

where $\mathrm{w}, \mathrm{d}$, and $\mathrm{h}$ represent the width, depth, and height respectively which are shown in Figure 3.1.

Because the dynamics of this model are in terms of the body coordinate system, this model needed to keep track of the orientation of the space object with respect to the inertial frame. To do so, every iteration of the function would take in the unit body frame vectors and translate it into the inertial frame using the direction cosine matrix (DCM) which could be constructed from the quaternion in the current state vector. The body coordinate unit vectors would be divided by the attitude matrix to produce unit normal vectors using Equation (3.1). The inertial unit vectors were also used to keep track of which facet of the cuboid was facing towards the sun and which of those were not at every time instance. If for any side the angle between the surface normal and the Sun's direction was larger than $\pi / 2$, than the solar radiation pressure would be set to zero since there was no radiant flux on that facet.

\subsubsection{Quaternions in UKF}

Applying the UKF structure to attitude estimation techniques demonstrated some shortcomings with the filtering techniques. Inherently, three parameter sets such as classical Euler angles or yaw, pitch, and roll sequences possess singularities. Thus to overcome this issue in the attitude parametrization, the quaternion representation was used. However, the limitations this representation presents is that quaternions 
are not additive and thus when calculated for the covariance matrix they needed manipulation. Reference [3] overcame this challenge by employing generalized Rodriguez parameters (GRPs), a three parameter set, to define the local error and then transforming those into quaternions, a four parameter set, for the global attitude.

The global attitude information in this work was parametrized using quaternions. As the name implies, quaternions are made up of four components which include a vector component and a scalar component [4]

$$
\{\boldsymbol{\varrho}\}=\left\{\begin{array}{l}
q_{1} \\
q_{2} \\
q_{3} \\
q_{4}
\end{array}\right\}=\left\{\begin{array}{l}
\mathbf{q} \\
q_{4}
\end{array}\right\}
$$

where $\mathbf{q}$ is the vector part $\left(\mathbf{q}=q_{1} \hat{\mathbf{i}}+q_{2} \hat{\mathbf{j}}+q_{3} \hat{\mathbf{k}}\right)$ and $q_{4}$ is the scalar part. The norm $\|\boldsymbol{\varrho}\|$ of the quaternion is defined as

$$
\|\varrho\|=\sqrt{\mathbf{q} \cdot \mathbf{q}+q_{4}^{2}}=\sqrt{q_{1}^{2}+q_{2}^{2}+q_{3}^{2}+q_{4}^{2}} .
$$

Considering unit quaternions, such that $\|\varrho\|=1$, the vector part and scalar part can be defined in terms of the principal angle of rotation, $\theta$, such that

$$
\mathbf{q}=\sin \frac{\theta}{2} \hat{\mathbf{u}} \quad q_{4}=\cos \frac{\theta}{2}
$$

Once the state vector had a specified quaternion and was fed into the UKF, the local error could be represented by the GRPs. A vector of GRP can be determined through

$$
\boldsymbol{\delta} \boldsymbol{p}=f \frac{\boldsymbol{\delta} \varrho}{a+\delta q_{4}}
$$




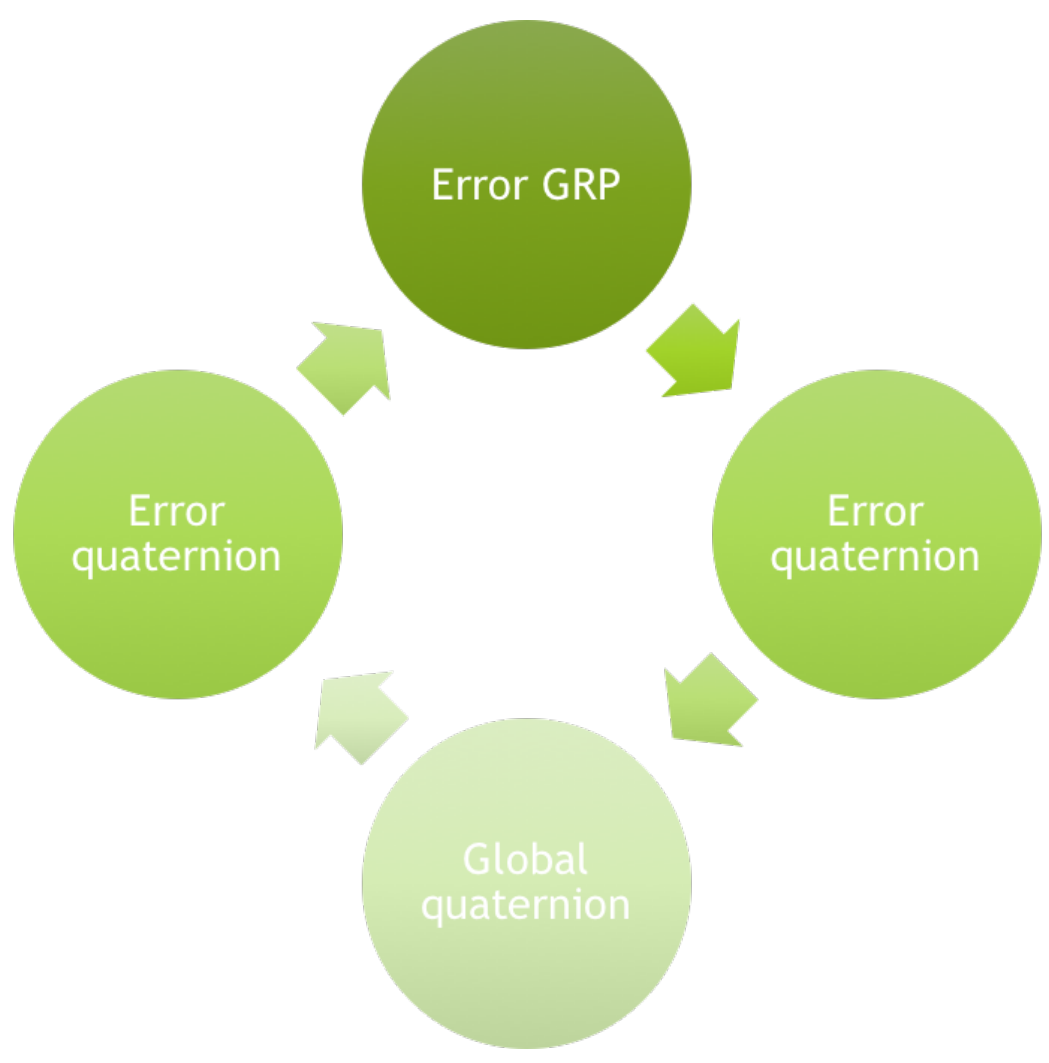

Figure 3.4: Error quaternion sequence for attitude implementation.

The representation of the attitude error as a GRP is useful for the propagation and updates states of the attitude covariance because the structure of the UKF can be used directly alongside the rest of the mechanics in the filter. In [3] the covariance matrix is interpreted as the covariance of the error GRP because for small angle errors, the error GRP is additive and the UKF structure can be used directly to compute sigma points. The error GRP sigma points are converted to error quaternions and then to global quaternions for the propagation stage. To compute the propagated covariance the global quaternions are converted to error quaternions and then back to error GRPs [12]. The process flow inside the UKF can be seen in Figure 3.4.

In order to carry out the process of manipulating the attitude error within the UKF, several transformations had to be done. The first was initializing the UKF with two separate state vectors for each iteration, one which carried the information 
of the error GRP state and one which carried the quaternion state vector information. These vectors were given by

$$
\hat{\mathbf{x}}_{k}^{\delta \mathbf{p}}=\left[\begin{array}{c}
\hat{\mathbf{r}}^{I} \\
\hat{\mathbf{v}}^{I} \\
\boldsymbol{\delta} \mathbf{p} \\
\hat{\boldsymbol{\omega}}_{B / I}^{B} \\
A \\
m
\end{array}\right] \quad \hat{\mathbf{x}}_{k}^{\varrho}=\left[\begin{array}{c}
\hat{\mathbf{r}}^{I} \\
\hat{\mathbf{v}}^{I} \\
\boldsymbol{\varrho}_{I}^{B} \\
\hat{\boldsymbol{\omega}}_{B / I}^{B} \\
A \\
m
\end{array}\right]
$$

where $\boldsymbol{\delta} \mathbf{p}$ is the error GRP state associated with the quaternion $\boldsymbol{\varrho}_{I}^{B}$. Upon first iteration, the initial estimate of the mean sigma point is denoted $\boldsymbol{\chi}_{0}(0)$. The error GRP of this initial state is set to zero, while the other states are initialized by their respective estimates.

The vector containing the error GRP is by design dimension $m-1$ if the state vector containing the quaternion is dimension $m$. Then to construct the error quaternion, denoted by $\boldsymbol{\delta} \varrho_{k}^{-}(i)$, associated with the $i^{\text {th }}$ error GRP sigma point is computed as

$$
\begin{gathered}
\delta q_{4_{k}}^{-}=\frac{-a\left\|\boldsymbol{\chi}_{k}^{\delta p}(i)\right\|^{2}+f \sqrt{f^{2}+\left(1-a^{2}\right)\left\|\chi_{k}^{\delta p}(i)\right\|^{2}}}{f^{2}+\left\|\boldsymbol{\chi}_{k}^{\delta p}(i)\right\|^{2}} \\
\boldsymbol{\delta} \mathbf{q}_{k}^{-}=f^{-1}\left[a+\delta q_{4_{k}}^{-}\right] \boldsymbol{\chi}_{k}^{\delta p}(i) \\
\boldsymbol{\delta} \boldsymbol{\varrho}_{k}^{-}(i)=\left[\begin{array}{c}
\boldsymbol{\delta} \mathbf{q}_{k}^{-} \\
\delta q_{4_{k}}^{-}
\end{array}\right]
\end{gathered}
$$

where $f=2(a+1), a$ is a parameter between 0 and 1 , and $\left\|\chi_{k}^{\delta p}(i)\right\|^{2}$ is the norm of the $i^{\text {th }}$ sigma point. $\chi_{k}^{\delta p}(i)$ should be a 3 by 1 vector of sigma points to allow the GRP sigma points to be turned into the error quaternion. The error quaternion must 
then be turned into a sigma point quaternion to be properly propagated through the equations of motion using

$$
\hat{\boldsymbol{\varrho}}_{k}^{-}(i)=\boldsymbol{\delta} \hat{\boldsymbol{\varrho}}_{k}^{-}(i) \otimes \hat{\boldsymbol{\varrho}}_{k}^{-}(0)
$$

where

$$
\mathbf{q}^{\prime} \otimes \mathbf{q}^{\prime \prime}=\left[\Psi\left(\mathbf{q}^{\prime}\right) \quad \mathbf{q}^{\prime}\right] \mathbf{q}^{\prime \prime}
$$

which becomes

$$
\left[\begin{array}{l}
q_{1} \\
q_{2} \\
q_{3} \\
q_{4}
\end{array}\right]=\left[\begin{array}{cccc}
q_{4}^{\prime} & -q_{3}^{\prime} & q_{2}^{\prime} & q_{1}^{\prime} \\
q_{3}^{\prime} & q_{4}^{\prime} & -q_{1}^{\prime} & q_{2}^{\prime} \\
-q_{2}^{\prime} & q_{1}^{\prime} & q_{4}^{\prime} & q_{3}^{\prime} \\
-q_{1}^{\prime} & -q_{2}^{\prime} & -q_{3}^{\prime} & q_{4}^{\prime}
\end{array}\right]\left[\begin{array}{l}
q_{1}^{\prime \prime} \\
q_{2}^{\prime \prime} \\
q_{3}^{\prime \prime} \\
q_{4}^{\prime \prime}
\end{array}\right]
$$

This global quaternion is then propagated through the equations of motion to get a propagated state vector and the process is reversed. The global propagated quaternion is to a local error quaternion by

$$
\hat{\boldsymbol{\varrho}}_{k+1}^{-}(i)=\boldsymbol{\delta} \varrho_{k+1}^{-}(i) \otimes\left[\hat{\varrho}_{k+1}^{-}(0)\right]^{-1}
$$

and then that can be turned into an error GRP by a variant of Equation (3.24),

$$
\boldsymbol{\delta} \boldsymbol{p}_{k+1}^{-}(i)=f \frac{\boldsymbol{\delta} \hat{\boldsymbol{q}}_{k+1}^{-}(i)}{a+\delta \hat{q}_{4_{k+1}}^{-}(i)}
$$

The process will then start over as the updated states become the initial states and the GRP error is reset to zero. Further details are provided in Section 3.6.3 in conjunction with Tables 3.1 and 3.2.

\subsubsection{Unscented Kalman Filter Algorithm}

Tables 3.1 and 3.2 detail the algorithm used for the UKF in this work. The filter begins with defining two initial state vectors, one that includes quaternion states and one that includes error GRP states as seen in Equation (3.25). The initial covariance matrix 
is defined as the initial error covariance for the state vector that includes the GRP states, the translational, rotational and parametric states. This is then used to form error GRP sigma points. The error GRP sigma points are converted to quaternion sigma points by creating error quaternions from each error GRP and then multiplying the error quaternion to the initial mean quaternion using Equation (3.27). This state vector is then passed through the translational dynamics of the model as modeled by Equation (3.19). The propagated mean sigma point quaternion, $\hat{\mathfrak{\varrho}}_{k+1}^{-}(0)$, is computed and stored, and error quaternions corresponding to each propagated quaternion sigma point are computed [12]. In the time update step, the propagated mean and covariance are calculated as a weighted sum of the sigma points. A measurement update is calculated using the model dynamics described in Equation (3.13). From there cross and innovations covariances are computed using the sigma point values. Next, the Kalman gain is calculated and used to update the mean and covariance vectors that contain the error GRPs. And finally, the quaternion update is found using Equation (3.26) which is then multiplied with the propagated mean quaternion. Since the error quaternion is now calculated, the error GRP can be reset to zero for the next process to continue. This updated state vector and covariance matrix is then passed out of the filter and recorded before another time iteration passes.

\subsection{Mass Calculation}

The primary focus of this work was to formulate a tool for mass calculation of a space object and doing so required some assumptions and simplifications. These included the geometry of the space object being a cuboid and assuming the object is a rigid body. One of the largest translational assumptions was attributing the entire deviation of the position vector solely to solar radiation pressure perturbations. Once the UKF and truth state vectors were calculated the value of SRP force that disturbed 
Table 3.1: UFK steps, 1 of 2

\begin{tabular}{|c|c|}
\hline $\begin{array}{c}\text { Initialize with } \\
\hat{\mathbf{x}}_{0}^{\delta \mathbf{p}}=E\left\{\mathbf{x}_{0}^{\delta \mathbf{p}}\right\} \\
\hat{\mathbf{x}}_{0}^{\varrho}=E\left\{\mathbf{x}_{0}\right\} \\
\mathbf{P}_{0}^{\delta \mathbf{p}}=E\left\{\left(\mathbf{x}_{0}^{\delta \mathbf{p}}-\hat{\mathbf{x}}_{0}^{\delta \mathbf{p}}\right)\left(\mathrm{x}_{0}^{\delta \mathbf{p}}-\hat{\mathbf{x}}_{0}^{\delta \mathbf{p}}\right)^{T}\right\}\end{array}$ & \\
\hline 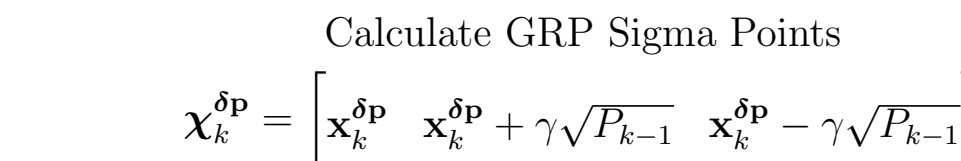 & \\
\hline $\begin{array}{c}\text { Calculate Quaternion Sigma Points } \\
\chi_{k}^{\varrho}=\left[\begin{array}{lll}\mathbf{x}_{k}^{\varrho} & \mathbf{x}_{k}^{\varrho}+\gamma \sqrt{P_{k-1}} & \mathbf{x}_{k}^{\varrho}-\gamma \sqrt{P_{k-1}}\end{array}\right]\end{array}$ & \\
\hline $\begin{array}{l}\text { Propagate Quaternion Sigma Points } \\
\qquad \chi_{k}^{\varrho}=\mathbf{f}\left[\chi_{k-1}^{\varrho}, t\right]\end{array}$ & \\
\hline 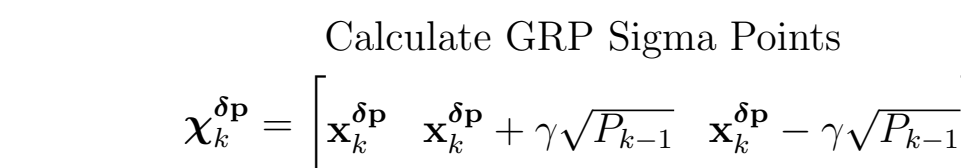 & \\
\hline $\begin{array}{c}\text { Time Update } \\
\hat{\mathbf{x}}_{k+1}^{-}=\sum_{i=0}^{2(L-1)} W_{i}^{\text {mean }} \boldsymbol{\chi}_{k+1}(i) \\
P_{k+1}^{-}=\sum_{i=0}^{2(L-1)} W_{i}^{\operatorname{cov}}\left[\boldsymbol{\chi}_{k+1}(i)-\hat{\mathbf{x}}_{k+1}^{-}\right]\left[\boldsymbol{\chi}_{k+1}(i)-\hat{\mathbf{x}}_{k+1}^{-}\right.\end{array}$ & $1]^{T}+Q_{K+1}$ \\
\hline
\end{tabular}


Table 3.2: UFK steps, 2 of 2

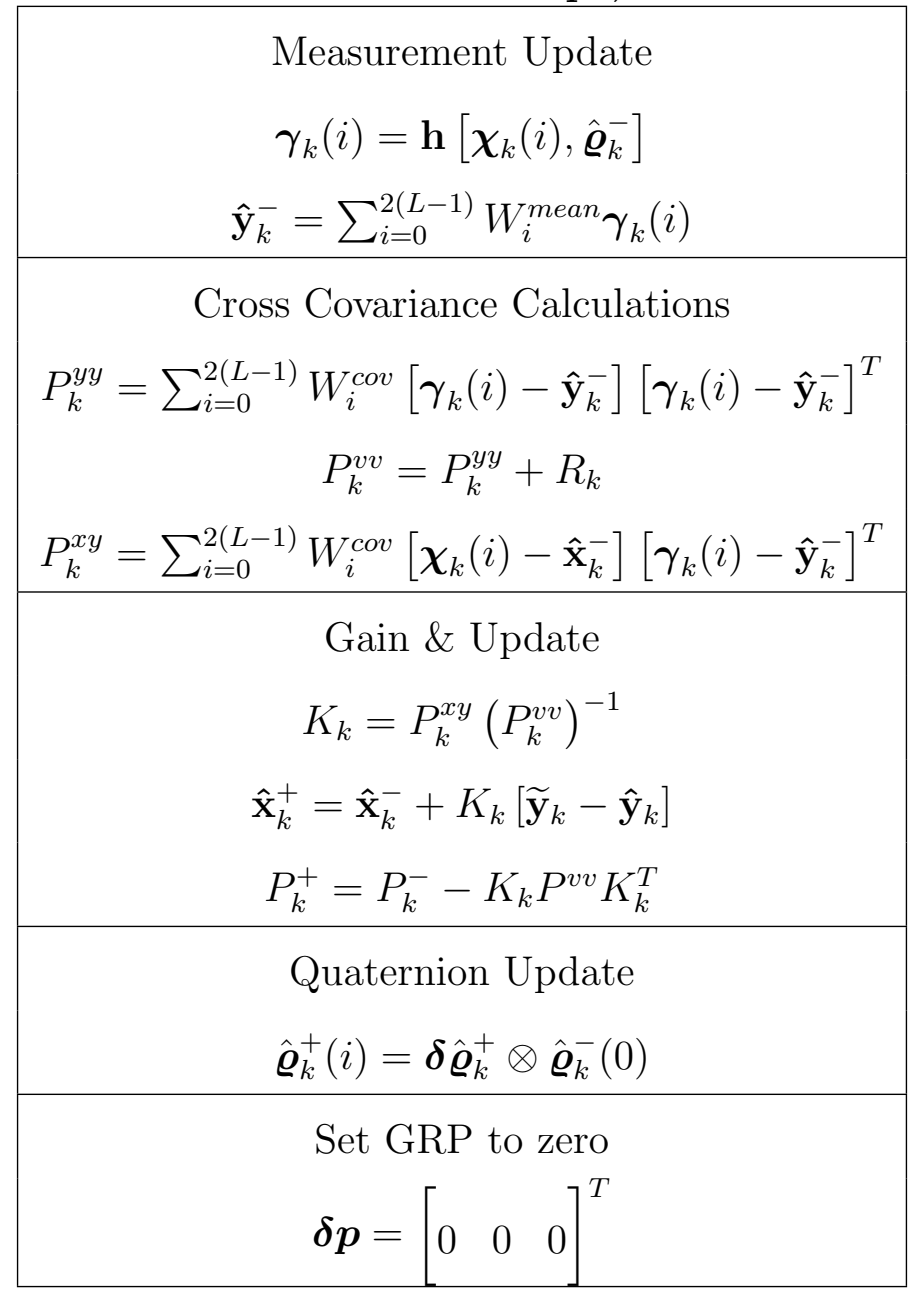


the vector by some difference, $\delta \mathbf{r}$, was calculated and stored for every time point that the state vector was calculated at. Then this value of $\mathbf{a}_{s r p}^{I}$ was used in conjunction with Equation (3.5) to solve for the area-to-mass ratio, $\frac{C_{r} A}{m}$ for each flat plate of the space object. Being that the space object was assumed to be a cuboid with six identical flat plate facets, reference [12] shows that $C_{r}$ can be approximated to be $C_{r}=1+\frac{4}{9} C_{d i f f}$ where $C_{d i f f}$ is the coefficient of diffusion. For this application, $C_{d i f f}=a$ where $a$ is the albedo of the flat plate. This assumption is valid because it is assumed the space object is completely diffuse and follows Lamberts cosine law. Thus the mass is able to be extracted from the area-to-mass ratio using the propagated albedo-area vector, $\mathbf{A C}_{\text {diff }}$ from the state vector. The benefit of this approach is that since the albedo-area vector is part of the estimated state vector the orientation of the space object is not affected by the uncertainty in the assumed albedo, but rather the assumed albedo is used to compute the SRP force on the space object and consequently the mass. 


\section{CASE STUDY DESIGN \& ANALYSIS}

The data analysis was set up in a case study format to make sure the code worked consistently with three different regimes: geosynchronous (GEO), highly elliptic (HEO), and medium earth orbit (MEO). The focus was on the different orbital altitudes so that consistency could be shown applying this to mass estimation techniques used in any debris or unknown space object collection application, however the focus of this paper was to ultimately have the application be to HAMR objects in the GEO regime. Each case study had a different way of initializing the orbit position and velocity vectors, both of which are discussed in Section 3.1. The information that stayed consistent throughout all cases was:

1. Time span for the UKF filter to run, 3 days in 30 second time intervals

2. Optical site was located at $-119^{\circ} 25^{\prime} 5^{\prime \prime}$ East longitude, $36.778^{\circ}$ latitude, $0 \mathrm{~km}$ above sea level

3. Collection time started April 20th, 2016 at 2:30am

4. The dimensions of the cuboid were $d=4 m, w=4 m, h=8 m$

5. The initial quaternion was $\varrho_{I}^{B}=\left[\begin{array}{llll}-\frac{1}{\sqrt{2}} & 0 & 0 & \frac{1}{\sqrt{2}}\end{array}\right]^{T}$

6. The initial orientation was achieved by rotating the cuboid $90^{\circ}$ about the $\mathrm{x}$-axis from its position described in Figure 3.1

7. The initial angular velocity was $\boldsymbol{\omega}_{B / I}^{B}=\left[\begin{array}{lll}0 & 0.003 & 0\end{array}\right]^{T}$

8. The albedo $a=0.3$ for every facet which meant that $C_{d i f f}=0.3$ as well because this is a typical value for satellite material 
Since the optical site was chosen to be in California, USA the case study targets were chose such that they would be visible from the site during the middle of the night to early morning. Section 3.5 explains how the effect of the Earth's shadow was accounted for in the SRP calculations while the space objects were propagated for 3 days of orbit. The angles data used for the initial orbit determination sections was generated by hand using the same optical site information the observations would be taken from and choosing three distinct points within a small orbital arc that was propagated using Vallado's SGP4 propagator [14].

The error for the state vector values was also consistent between trials. For the position the error was set to be $20^{2} \mathrm{~km}^{2}$, the velocity error was $0.01^{2}(\mathrm{~km} / \mathrm{s})^{2}$, and $5^{\circ}$ for all three attitudes. The angular velocity error was $0.001(\mathrm{rad} / \mathrm{s})^{2}$, each area and albedo area of each face of the cuboid had a $10^{2} \mathrm{~m}^{4}$ error and the mass had a $3^{2} \mathrm{~kg}^{2}$ error. The truth mass for each satellite was the actual value of the known space object. With the area estimations this gave area to mass ratios ranging from $0.0053-0.058 \frac{\mathrm{m}^{2}}{\mathrm{~kg}}$. While these values are not in the range of being classified a HAMR object, the results provide valuable information for applications to HAMR data once it can be collected and run through the tool.

The figures that follow, $4.1-4.6$, are all visually represent the difference between the UKF measurement and the truth value. The 'truth' vector is found using all the same equations of translational and rotational motion as well as the same measurement equations for flux but rather than being propagated through the UKF it is propagated separately through ODE45 solver built into MATLAB. The result allows comparison to what the UKF tool has estimated the values to be and what the values of a propagated state would be. Thus it is important to see in the following figures that the value 0 is included within the $3 \sigma$ error indicating no difference between estimated and truth. This is true for every graph except (c) for the magnitude to which it is a simple apparent magnitude measurement recorded, not shown as a difference 
to the truth value. The magnitude measurements were found after the UKF was run found by taking the flux measurements at each time step and using Equation (3.14) were transformed into an apparent magnitude. This was done for both the estimate values and the error which can account for the non-symmetrical nature of the magnitude $3 \sigma$ error present in all the (c) graphs to follow. For more detailed views of the following figures please refer to Appendix A.

\subsection{Case 1: GEO}

\subsubsection{TLE}

The object chosen for this study was Galaxy 15, SSN: 28884. Galaxy 15 features a unique commercial/government hybrid payload configuration and thus has functionality for American telecommunications broadcasting and GPS data applications. As can be seen from Figure 4.1a, the position values have converging $3 \sigma$ error bounds as the difference values oscillate around a mean of 0 . Figure $4.1 \mathrm{~b}$ depicts the velocity deviation from the truth value and shows a tending toward the value of 0 , but the errors seem to grow before converging toward 0 . The magnitude stays at a consistent value of about 10th magnitude seen in Figure 4.1c which is common for a GEO satellite of this size and shape. And finally, the mass value can be seen to be calculated very accurately with few variations around the 0 mean, shown in Figure 4.1d. This was compared to the actual mass value of $885 \mathrm{~kg}$ [16]. The issue with this result is the diverging mass estimate value from the UKF which will be discussed later in Section 5. 

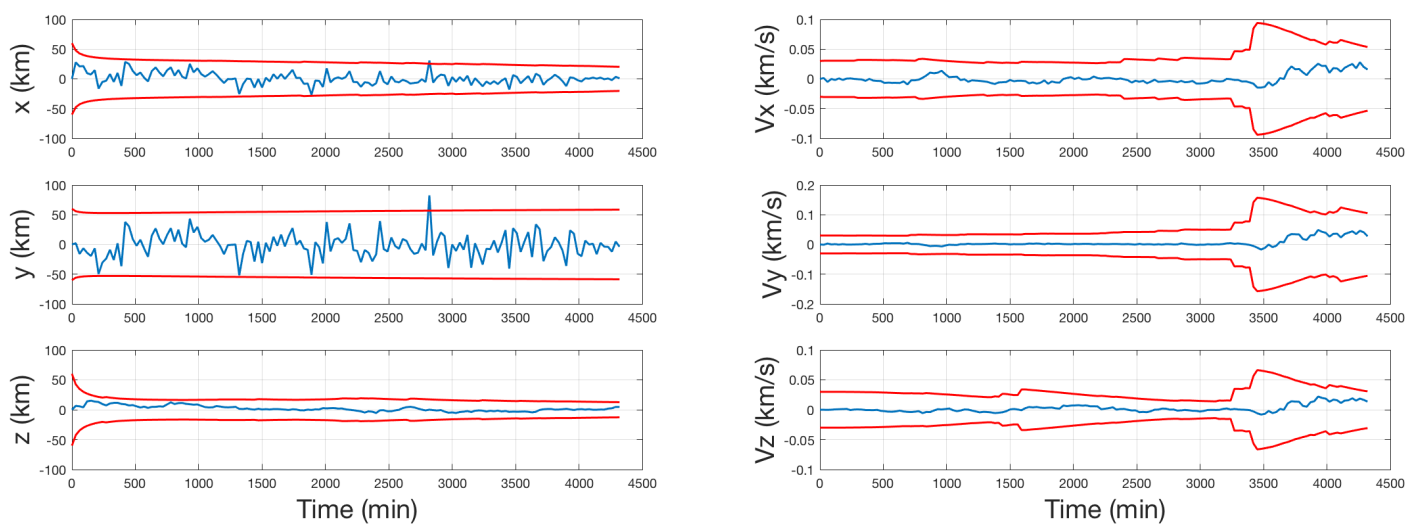

(a)

(b)
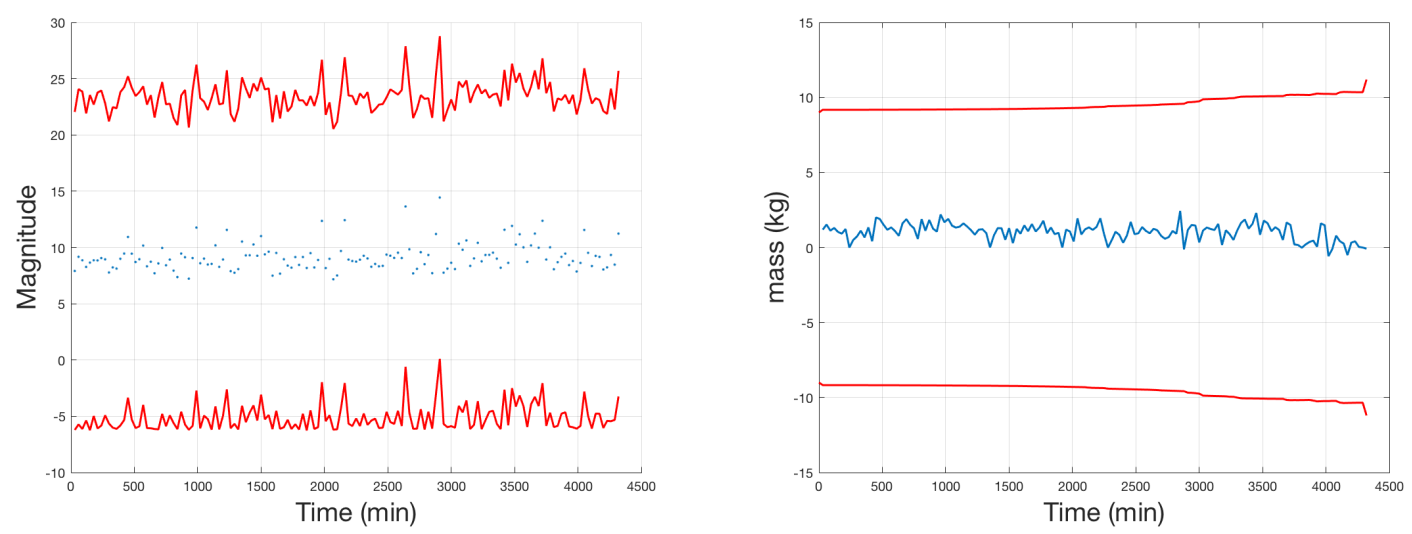

(c)

(d)

Figure 4.1: Satellite 28884 Estimate Results: (a) Position estimation with $3 \sigma$ error; (b) Velocity estimation with $3 \sigma$ error; (c) Apparent magnitude estimation with $3 \sigma$ error; and, (d) Mass estimation with $3 \sigma$ error. 


\subsubsection{IOD}

The object chosen for this study was ANIK F1-R, SSN: 28868. Anik F1-R provides telecommunications, broadcasting and Internet services over a large zone covering Canada and North America as well as it is equipped with a navigation payload. Figure 4.2 shows very similar results as Figure 4.1 did with a few exceptions. Being that the state vector was initialized from a set of angles only estimates the range data was not accurately determined like it could be in the case of using a TLE. Thus the $\mathbf{d}^{I}$ vector in Equation (3.13) is a guess which has error associated with it. For this reason the magnitude estimates are lower, settling around 5th magnitude. This is a error which should be considered with all UKF applications because the measurement values which are used for photometric flux will be lower causing the filter to make larger errors. The mass in Figure 4.2d is being compared to the actual mass of 3,015 $\operatorname{kg}[16]$.

\subsection{Case 2: HEO}

\subsubsection{TLE}

The object chosen for this study was CLUSTER II-FM8, SSN: 26464. Cluster IIFM8 is a collection of four spacecraft flying in formation around the earth, relaying detailed information about solar wind's affect on our planet in three dimensions. Since the HEO objects have highly elliptical orbits by design, the study was conducted at perigee to insure the closest approach of the satellite was viable from how it was designed. CLUSTER II-FM8 has a perigee value of 33,473.6 km. As can be seen from Figure 4.3a, the position values have converging $3 \sigma$ error bounds as the difference values oscillate around a mean of 0 . Figure $4.3 \mathrm{~b}$ depicts the velocity deviation from the truth value and shows a tending toward the value of 0 , but has momentary periods 

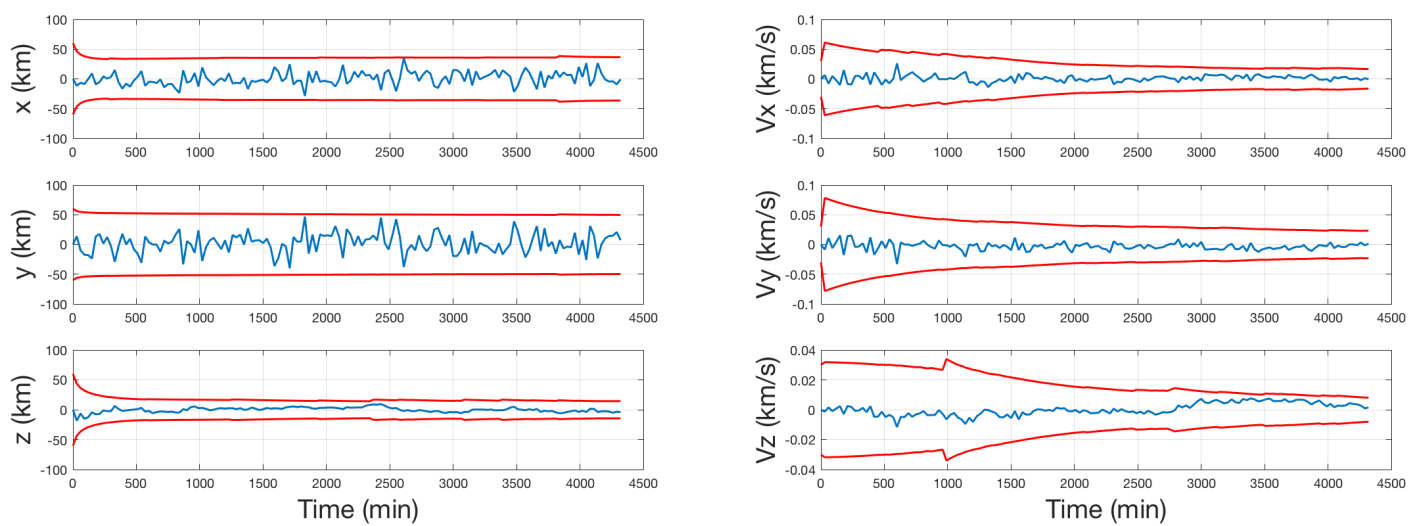

(a)

(b)
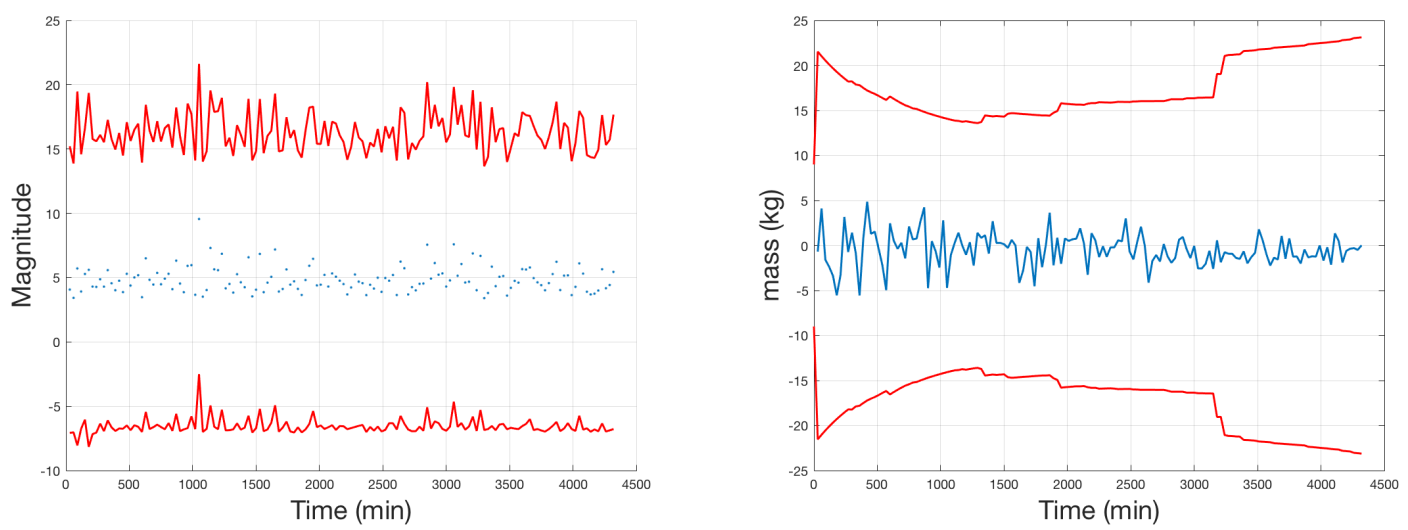

(c)

(d)

Figure 4.2: Satellite 28868 Estimate Results: (a) Position estimation with $3 \sigma$ error; (b) Velocity estimation with $3 \sigma$ error; (c) Apparent magnitude estimation with $3 \sigma$ error; and, (d) Mass estimation with $3 \sigma$ error. 

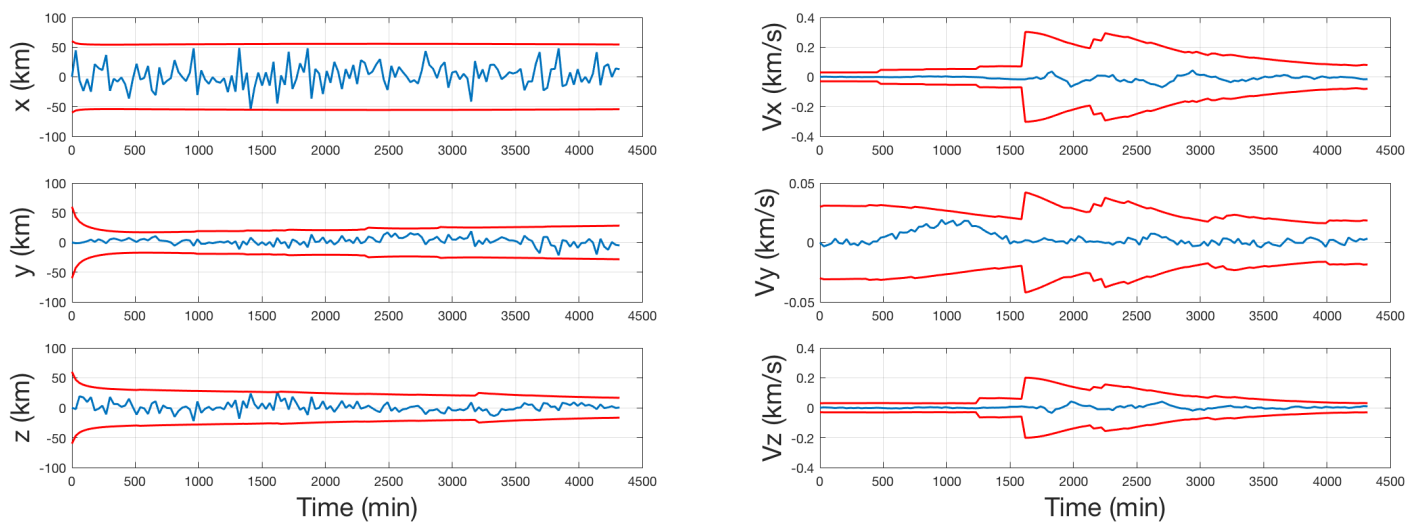

(a)

(b)
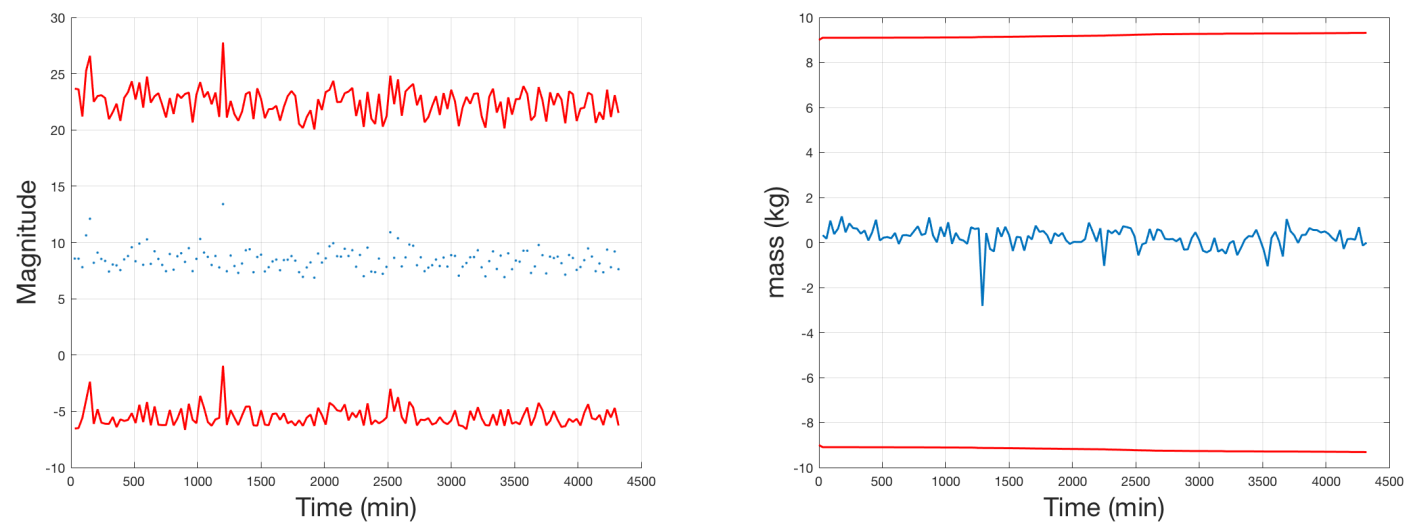

(c)

(d)

Figure 4.3: Satellite 26464 Estimate Results: (a) Position estimation with $3 \sigma$ error; (b) Velocity estimation with $3 \sigma$ error; (c) Apparent magnitude estimation with $3 \sigma$ error; and, (d) Mass estimation with $3 \sigma$ error.

of growing error. The magnitude stays at a consistent value of about 8th magnitude seen in Figure 4.1c which is a proper estimation for a portion of the highly elliptical orbit which the satellite is at an orbital distance less than about $35,000 \mathrm{~km}$. And finally, the mass value can be seen to be calculated very accurately with few variations around the 0 mean, shown in Figure 4.1d. This was compared with the true mass of $550 \mathrm{~kg}[16]$. 


\subsubsection{IOD}

The object chosen for this study was SIRIUS 2, SSN: 26483. SIRIUS 2 enables Sband digital radio broadcasts (music, news, and entertainment) directly or through urban relay stations to motorists in North America. This study was also done at perigee, when SIRIUS 2 was at $23,452.8 \mathrm{~km}$. The resulting graphs in Figure 4.4 prove conclusive similar to the GEO case study with similar issues with magnitude measurements due to the nature of angles data not including range information. The actual mass compared in Figure $4.4 \mathrm{~d}$ for Sirius 2 is $3,800 \mathrm{~kg}$ [16]. The issue with this result is again the diverging mass estimate value from the UKF which will be discussed in Chapter 5.

\subsection{Case 3: MEO}

\subsubsection{TLE}

The object chosen for this study was NAVSTAR 50, SSN: 26690. NAVSTAR 50 (USA 156) is an American GPS navigational spacecraft that was launched by a Delta 2 rocket. The results of Figure 4.5 are similar to the GEO and HEO case for position, velocity, and magnitude estimates which allows for consistency of the UKF between different regime types. The mass estimates are very sporadic and consistently tending outside of the $3 \sigma$ error bounds when compared to the true mass of 2,032 $\mathrm{kg}$ [16]. Potential reasons for the uncharacteristic mass estimation is the lower altitude orbit. This will further be discussed in Section 5.2.

\subsubsection{IOD}

The object chosen for this study was NAVSTAR 57, SSN: 28874. NAVSTAR 57 is also a GPS satellite similar to NAVSTAR 50 but has three more frequency channels 

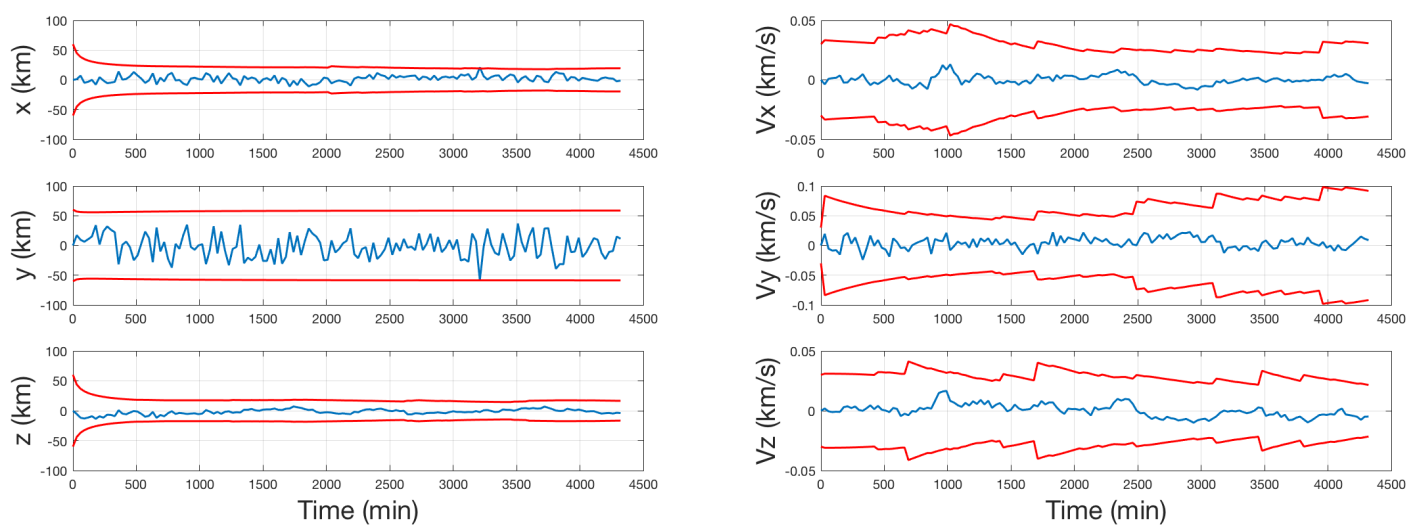

(a)

(b)
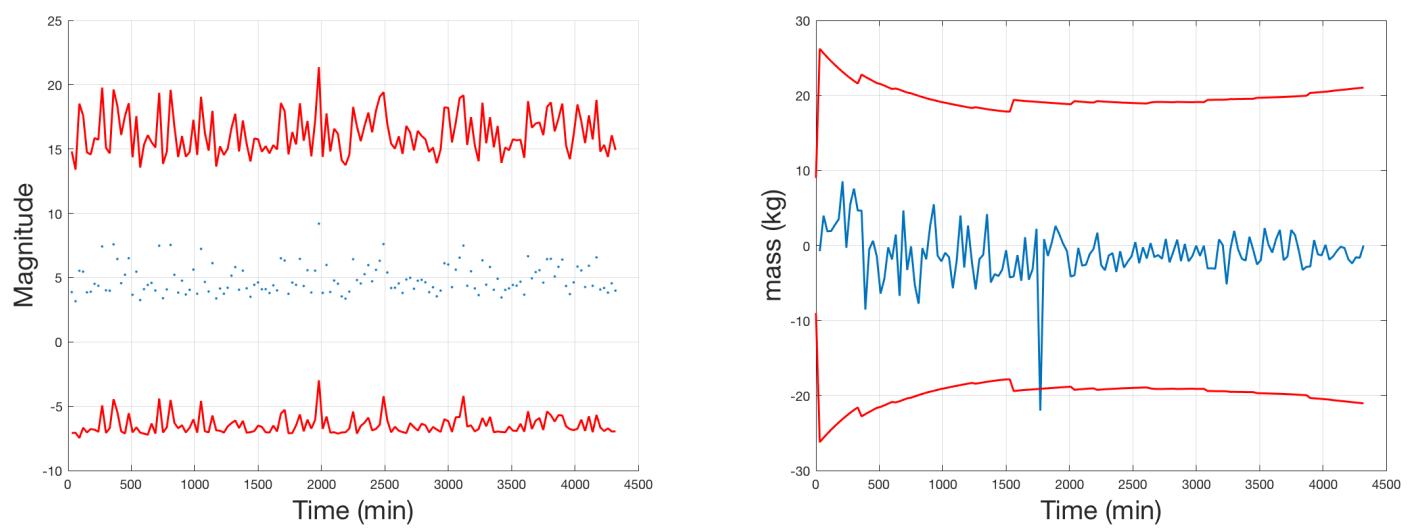

(c)

(d)

Figure 4.4: Satellite 26483 Estimate Results: (a) Position estimation with $3 \sigma$ error; (b) Velocity estimation with $3 \sigma$ error; (c) Apparent magnitude estimation with $3 \sigma$ error; and, (d) Mass estimation with $3 \sigma$ error. 

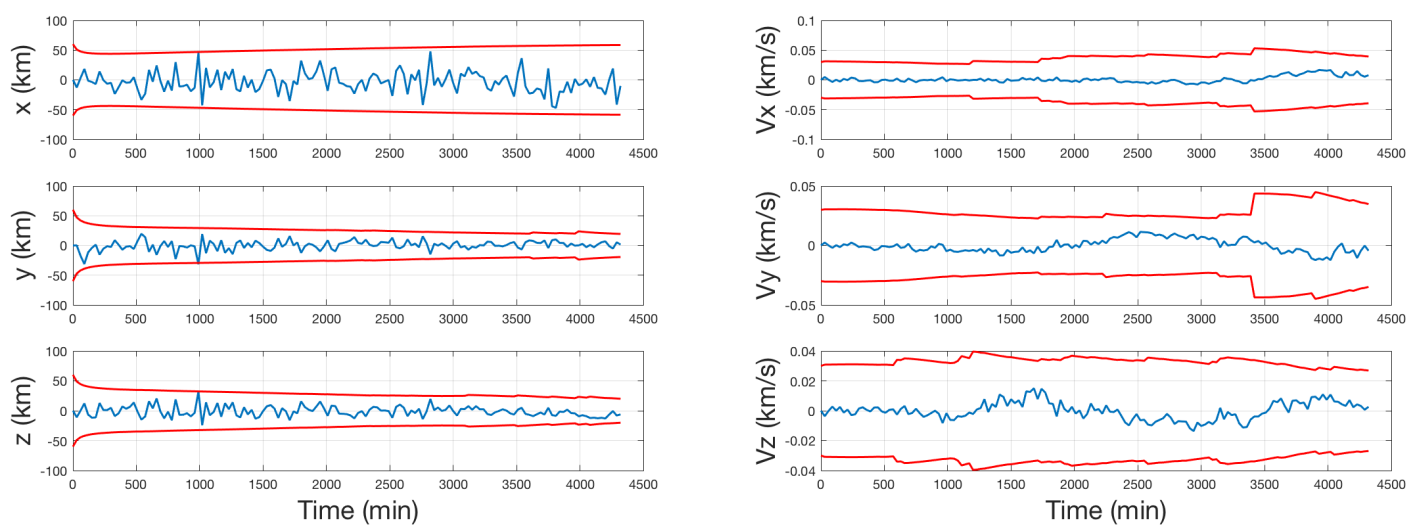

(a)

(b)
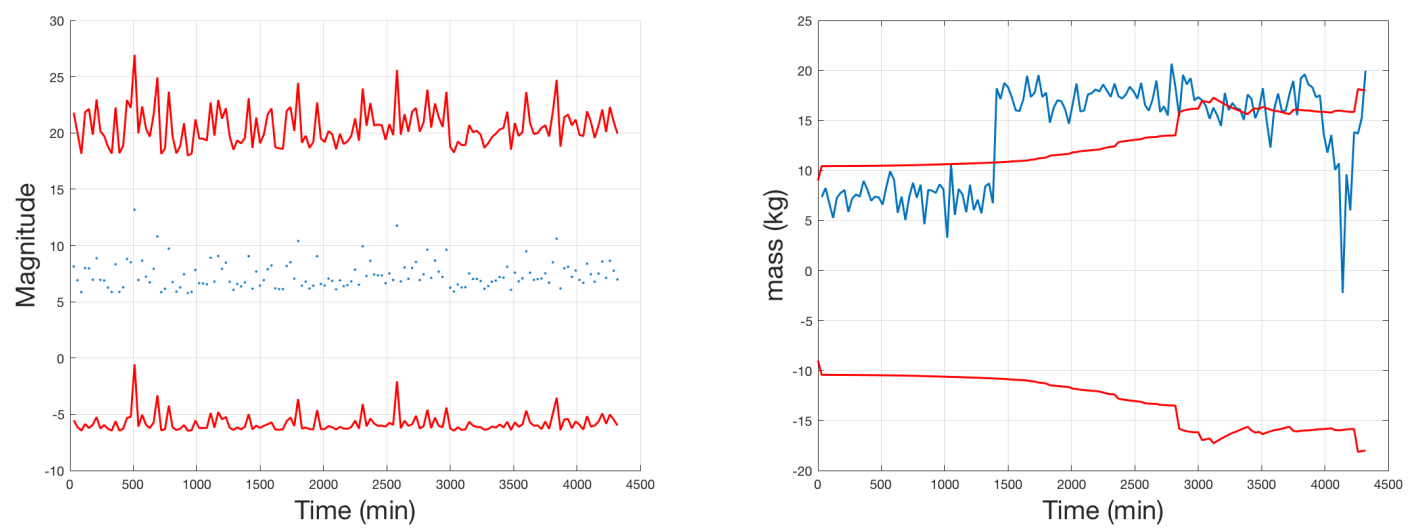

(c)

(d)

Figure 4.5: Satellite 26690 Estimate Results: (a) Position estimation with $3 \sigma$ error; (b) Velocity estimation with $3 \sigma$ error; (c) Apparent magnitude estimation with $3 \sigma$ error; and, (d) Mass estimation with $3 \sigma$ error. 
(with two more military and one more civilian), and is more secure against jamming and radiation than the older models. The position and velocity graphs in Figures 4.6a and 4.6b show strange characteristic errors which both converge and diverge from the mean of 0 but also have some values for the estimates outside the $3 \sigma$ error bounds which would cause hesitation to conclude this is a viable regime for the UKF to process. Figure 4.6d shows the true negative of using this tool for the MEO regime. The mass estimates are very sporadic and consistently tending outside of the $3 \sigma$ error bounds when compared to the true mass of 2,032 kg [16]. Again this could be due possibly to the lower altitude of the orbit. 

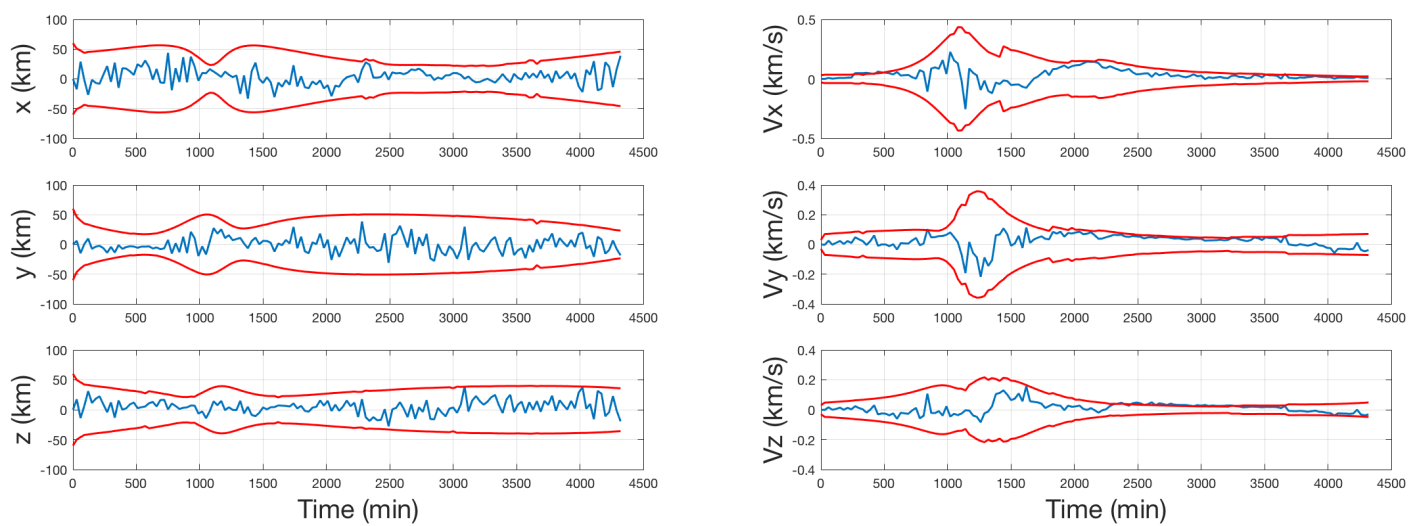

(a)

(b)
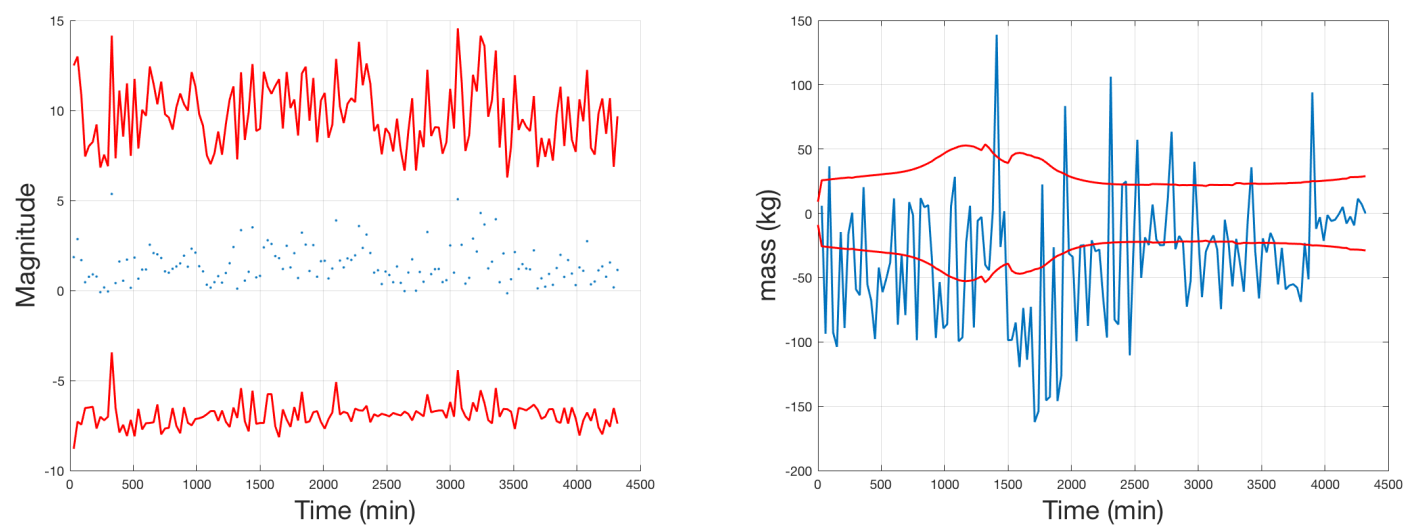

(c)

(d)

Figure 4.6: Satellite 28874 Estimate Results: (a) Position estimation with $3 \sigma$ error; (b) Velocity estimation with $3 \sigma$ error; (c) Apparent magnitude estimation with $3 \sigma$ error; and, (d) Mass estimation with $3 \sigma$ error. 


\section{CONCLUSIONS}

This thesis utilized an unscented Kalman filter formulation with light curve and angles data to estimate the the translational and rotational states of a space object along with the area, mass, and albedo area vector. The case study shown in Section 4 highlighted the differences between the two approaches of initializing the orbital data for the space object for three different regimes: GEO, HEO, and MEO. The application this has to HAMR objects is that it was shown the tool performs most accurately, based on error convergence and estimate accuracy, at higher regimes especially GEO. While this thesis did not actually have HAMR data to run through the estimator, the tool was built to take in data and process it proven by the robust estimations shown in this paper for known space objects. Therefore, going forward, data can be collected from a HAMR object in the GEO regime and run through this tool in order to extract information about the intrinsic properties of the object being observed. This will ultimately lead to cataloguing and more predictable orbit tracking of HAMR objects.

\subsection{Contributions}

The work done with this thesis contributed to the growing understanding of mass estimation methods for unknown space objects. Fusing astrometric and photometric data into a single tool which could be used for processing allows for more accurate area-to-mass ratio measurements and ultimately better dynamic modeling of the space object in question. As mentioned in Section 2.3, Linares et al. made a large

contribution to the community with their paper [12] but the tool and information was 
not made available to the public. It is my hope that the research published in this paper which attempted to reconstruct the formulation previously used for studying this information and make it available to be expanded upon and adapted to further methods of mass estimation. The hardest hurdle to surmount with HAMR object data collection is the short time span of data gathered. With integrating the tool to take in angles data for IOD as well as TLE information, it was my hope to adapt the tool to be a universal mass estimator that can be used in a variety of situations, from catalogued satellites such as those investigated in the case studies, or unknown space objects such as HAMR objects.

\section{$5.2 \quad$ Strengths and Weaknesses}

The tool performed remarkably at the higher altitude regimes, GEO and HEO, which by design was a desirable characteristic. HAMR objects are typically in the GEO regime and are causing threats to active RSOs. Being able to detect the objects and figure out the orbital path they are on with small amounts of data will allow for collision avoidance maneuvers to take place. The more accurate tools such as this can be, the better the HAMR object can be studied, catalogued, and tracked. With intrinsic property information such as mass and area estimates those tracking the HAMR objects have a better understanding of what it will do next to always stay one step ahead.

Once the space object under study reached about $20,000 \mathrm{~km}$ in altitude the UKF has some issues with the magnitude measurements and mass estimation values as can be seen in Figure 4.6d. This could be seen as a weakness to the tool but it came as the expense of modeling the proper dynamics for higher altitude space objects. Additionally, the incorporation of the IOD modeling for the space object's state caused magnitude estimations for the photometric data used in the Kalman filter 
which would hinder the results.

Some of the largest weaknesses come in the assumptions used to model the dynamics of the space object. The only disturbance perturbation incorporated into the translational dynamics is solar radiation pressure since this is the most dominate source of orbital change for these HAMR objects. However, other forces such as drag and Earth's oblateness could improve dynamics of the space object for estimation. Additionally, there are no disturbance torques incorporated into the rotational dynamics of the model but the tool has the capability to incorporate them at a later date since Euler's equation is modeled in the dynamics of Equation (3.19). Incorporating the dynamics for solar radiation in the rotational dynamics, the torques from Earth's magnetic field, and especially those from non-rigid body motion would be instrumental to better modeling the dynamics. HAMR objects whose origins have been able to be estimated have been found to be pieces of multi layered insulation from active satellites [11]. The effects of the aforementioned disturbance torques have not been studied much with HAMR objects and better make this tool more reliable. Additionally, not having to attribute all the error in the perturbed orbit to the SRP would most likely cause the divergence of the mass estimates seen in all of the (d) figures in Section 4 to instead converge with time. The could be other sources of error introduced at play here to cause this divergence but investigation into this assumption could be a great place to start.

The assumption of the space object shape, dimensions, and reflectively give this formulation another disadvantage. Being able to better estimate or even eliminate the coefficient of reflectively and albedo for the different facets of the space object would give a exponentially more accurate understanding to the area-to-mass ratio and mass estimation values. The albedo area and solar pressure albedo were assumed to be the same thing in this development. Unfortunately, these two quantities are only the same at zero phase angle or if the observer is standing on the sun, as discussed in 
Section 1.2. Disentangling the physics of these reflective properties would give more accurate estimated values of the mass for a particular space object of little known characteristics. And finally, a more detailed BRDF model could relate the difference between absorbed, diffuse, and specular reflectance better than assuming that the object is completely diffuse.

\subsection{Future Work}

The main purpose of this tool was developed to measure HAMR object mass however without actual HAMR data the results shown here were unable to test the validity to this application and instead had to measure performance with known space objects. Therefore it the most critical future work that could be implemented would be to acquire HAMR object data from multiple different nights of collects and run the tool to estimate the mass. This should be done on a well known HAMR object to be able to compare preliminary results with previous investigations into the intrinsic properties.

There are a number of applications which this tool can be built upon to improve the mass estimation as well as further investigation to the varying properties the tool can understand. For example, running this mass estimation tool and comparing the rate of convergence for dinnering area-to-mass ratios would be useful to know because the tool should be optimized for high area-to-mass ratios since that is the focus of the study. The faster and more efficient it could be at understanding HAMR objects, the better since most of the time the data collection is limited since these objects are captured with data from other GEO surveys.

The length of these case studies were only for a duration of 3 days. Being able to run the simulation for longer and produce more data would allow the UKF to converge to values that would hopefully be close and closer to the truth estimates. 
This was the motivation behind using the unscented formulation but the trade off between processing time and accuracy would have to be optimized to deliver the results the user is looking for.

Additionally, changing the tool to be able to not have albedo as a constant value, but instead be varying and estimated by the filter itself would prove very useful for applications where other properties such as area might be able to be deduced for the space object but then the albedo could be measured. The tool could be adapted to run simultaneously with different sets of information and ultimately characterize the space object in question with better accuracy.

Lastly, a sensitivity analysis would be very valuable and maybe lend insight in to some of the results. One could look at sensitivity to uncertainties in: data quantity, quality and cadence; area-to-mass ratio magnitude; or initial conditions to further understand the strengths and weaknesses of this tool when modeling dynamics of space objects and attempting to calculate mass estimates. 


\section{BIBLIOGRAPHY}

[1] M. Ashikhmin and P. Shirley. An anisotropic phong brdf model. Journal of graphics tools, 5(2):25-32, 2000.

[2] B. K. Bradley and P. Axelrad. Improved estimation of orbits and physical properties of objects in geo. In Advanced Maui Optical and Space Surveillance Technologies Conference (AMOS), Maui, HI, 2013.

[3] J. L. Crassidis and F. L. Markley. Unscented filtering for spacecraft attitude estimation. Journal of guidance, control, and dynamics, 26(4):536-542, 2003.

[4] H. D. Curtis. Orbital Mechanics for Engineering Students. Elsevier Aerospace Engineering Series. Elsevier, second edition, 2010.

[5] K. DeMars and M. Jah. Passive multi-target tracking with application to orbit determination for geosynchronous objects. AAS Paper, pages 09-108, 2009.

[6] K. J. DeMars. Flat plate and spherical object srp model equivalence. Acquired from Thomas M. Kelecy, August 2011.

[7] P. R. Escobal. Methods of orbit determination. New York: Wiley, 1965, 1965.

[8] M. Jah and R. A. Madler. Satellite characterization: angles and light curve data fusion for spacecraft state and parameter estimation. In Proceedings of the Advanced Maui Optical and Space Surveillance Technologies Conference, volume 49, 2007.

[9] M. Kaasalainen and J. Torppa. Optimization methods for asteroid lightcurve inversion: I. shape determination. Icarus, 153(1):24-36, 2001. 
[10] R. R. Karimi and D. Mortari. Initial orbit determination using multiple observations. Celestial Mechanics and Dynamical Astronomy, 109(2):167-180, 2011.

[11] T. Kelecy and M. Jah. Analysis of high area-to-mass ratio (hamr) geo space object orbit determination and prediction performance: Initial strategies to recover and predict hamr geo trajectories with no a priori information. Acta Astronautica, 69(7):551-558, 2011.

[12] R. Linares, M. K. Jah, J. L. Crassidis, F. A. Leve, and T. Kelecy. Astrometric and photometric data fusion for inactive space object mass and area estimation. Acta Astronautica, 99:1-15, 2014.

[13] T. Schildknecht, R. Musci, W. Flury, J. Kuusela, J. de Leon, and L. D. F. D. Palmero. Properties of the high area-to-mass ratio space debris population in geo. In 2005 AMOS Technical Conference Proceedings, Kihei, Maui, HI, 2005.

[14] D. Vallado and P. Crawford. Sgp4 orbit determination. In $A I A A / A A S$ Astrodynamics Specialist Conference and Exhibit, page 6770, 2008.

[15] D. A. Vallado and W. D. McClain. Fundamentals of Astrodynamics and Applications, volume The Space Technology Library. Microcosm Press, fourth edition, 2013.

[16] M. Wade et al. Encyclopedia astronautica. Mark Wade, 1999. 


\section{APPENDIX}

\section{DETAILED VIEW OF RESULT GRAPHS}

The following pages include a more detailed view of Figures $4.1-4.6$. 

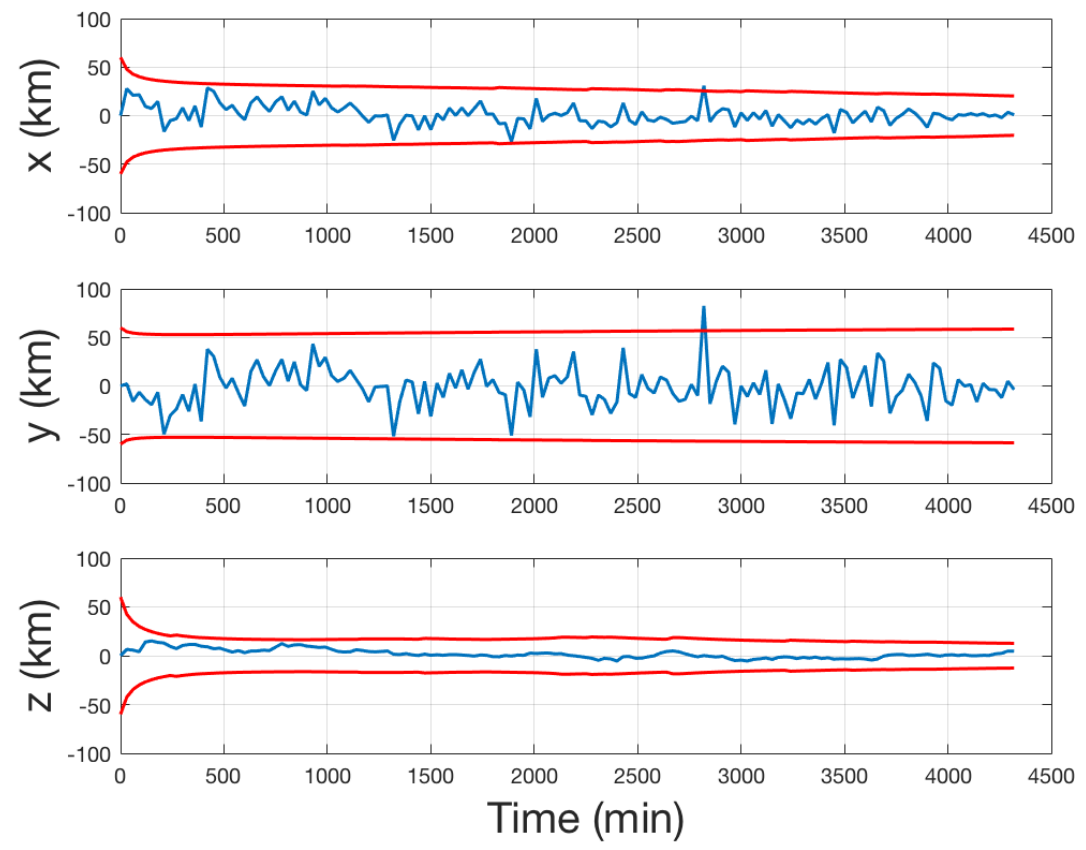

Figure A.1: Satellite 28884 position estimate with $3 \sigma$ error bounds.
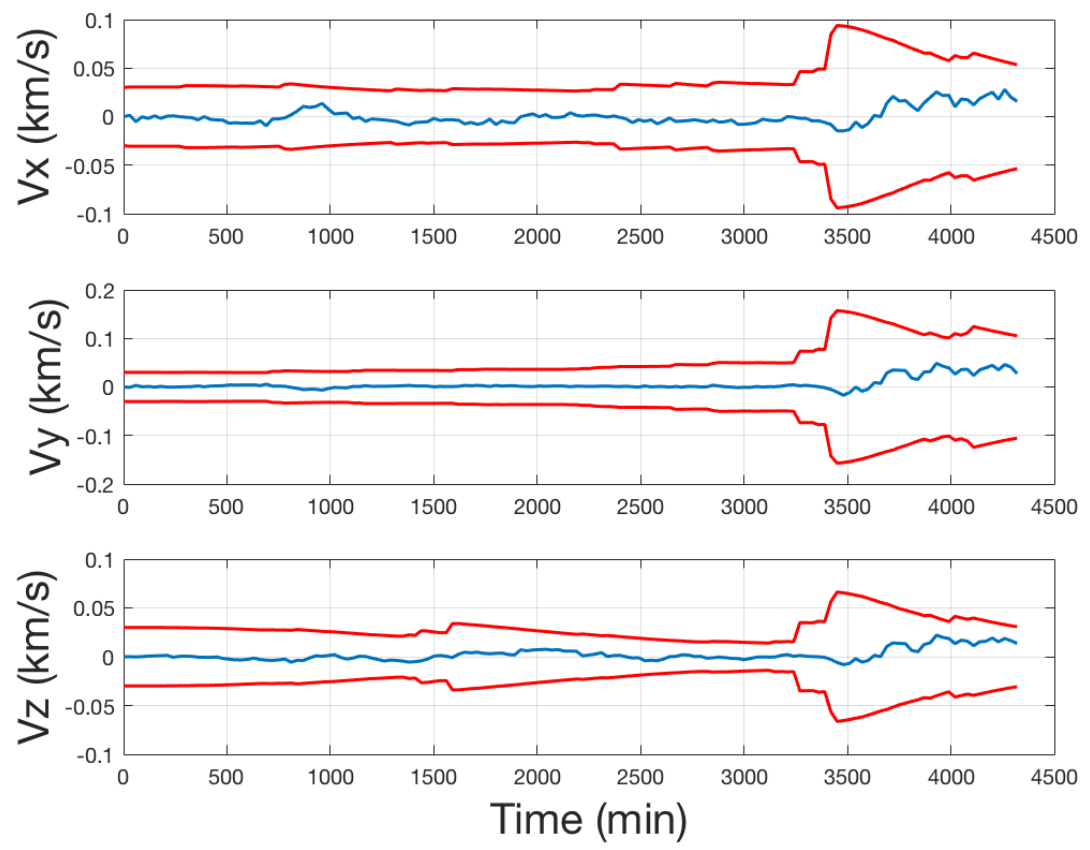

Figure A.2: Satellite 28884 velocity estimate with $3 \sigma$ error bounds. 


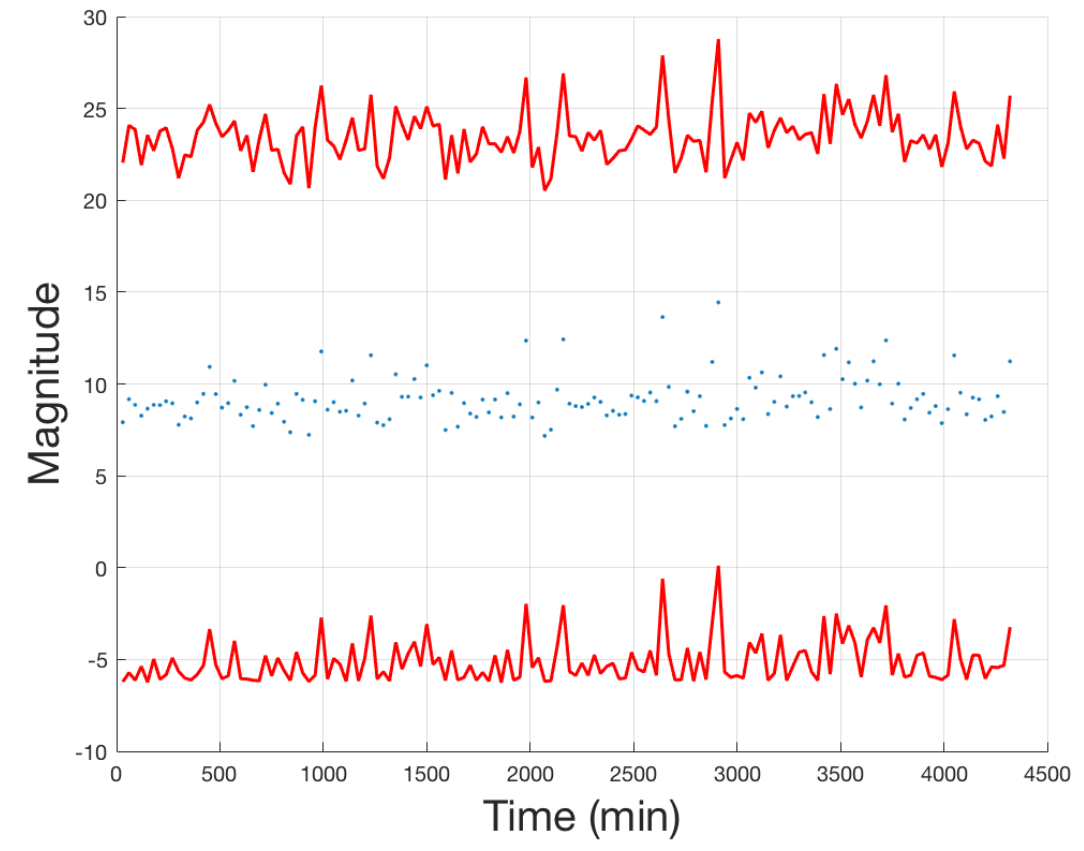

Figure A.3: Satellite 28884 apparent magnitude estimate with $3 \sigma$ error bounds.

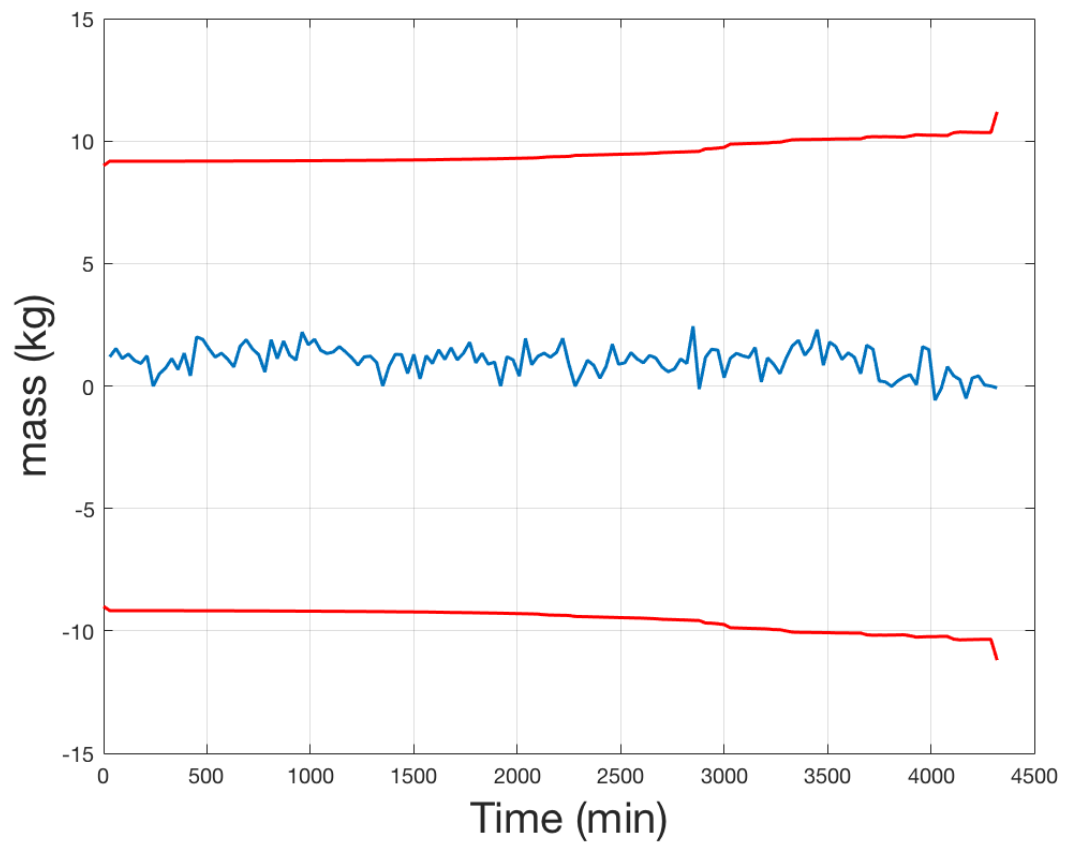

Figure A.4: Satellite $\mathbf{2 8 8 8 4}$ mass estimate with $3 \sigma$ error bounds. 

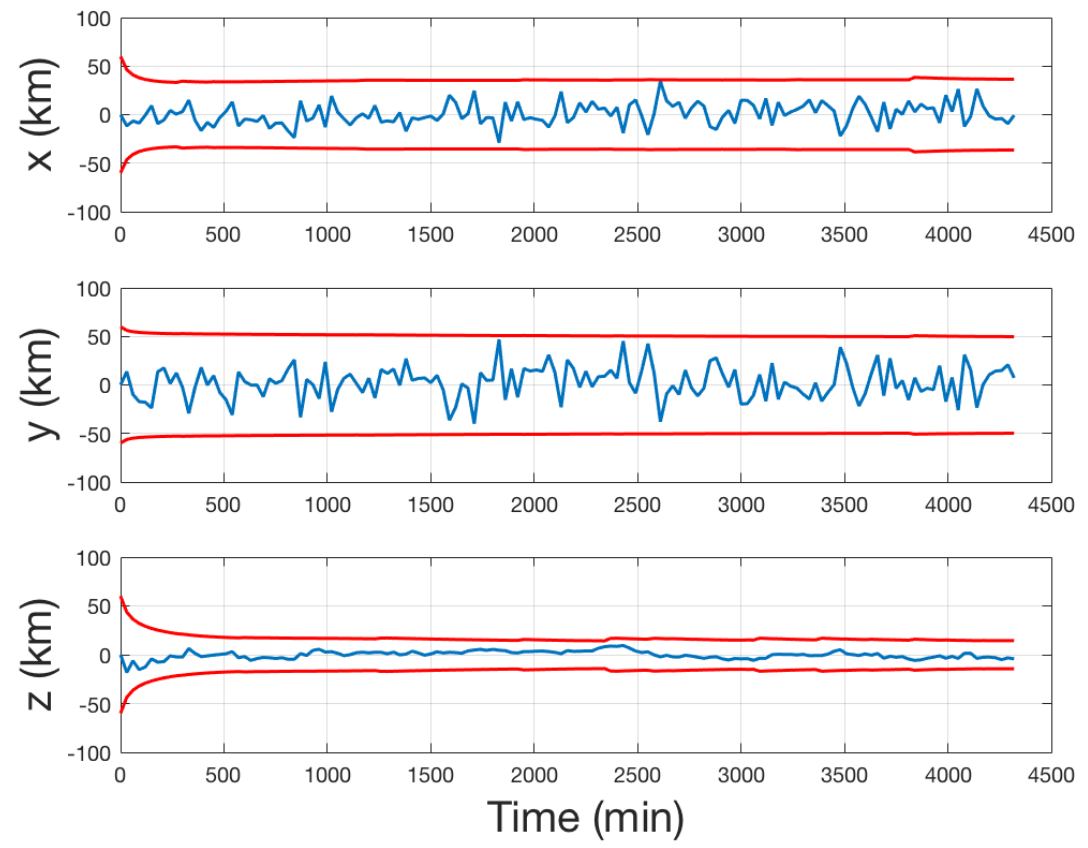

Figure A.5: Satellite 28868 position estimate with $3 \sigma$ error bounds.
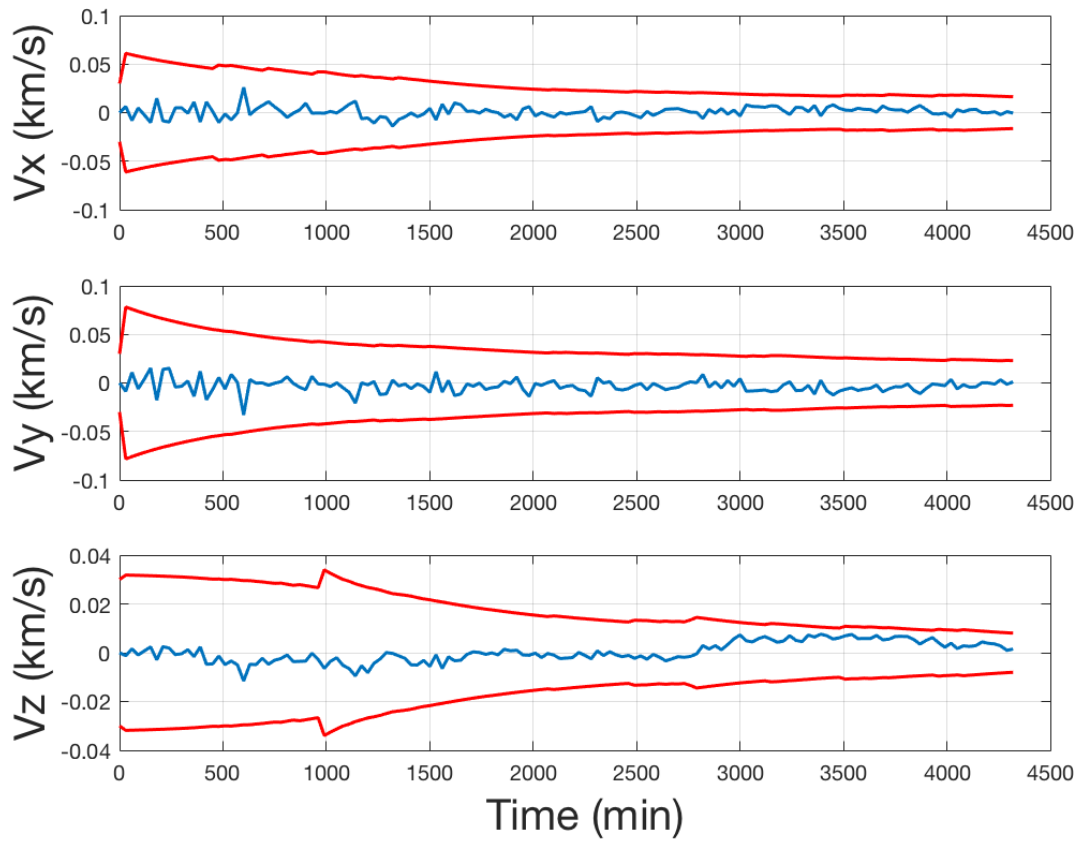

Figure A.6: Satellite 28868 velocity estimate with $3 \sigma$ error bounds. 


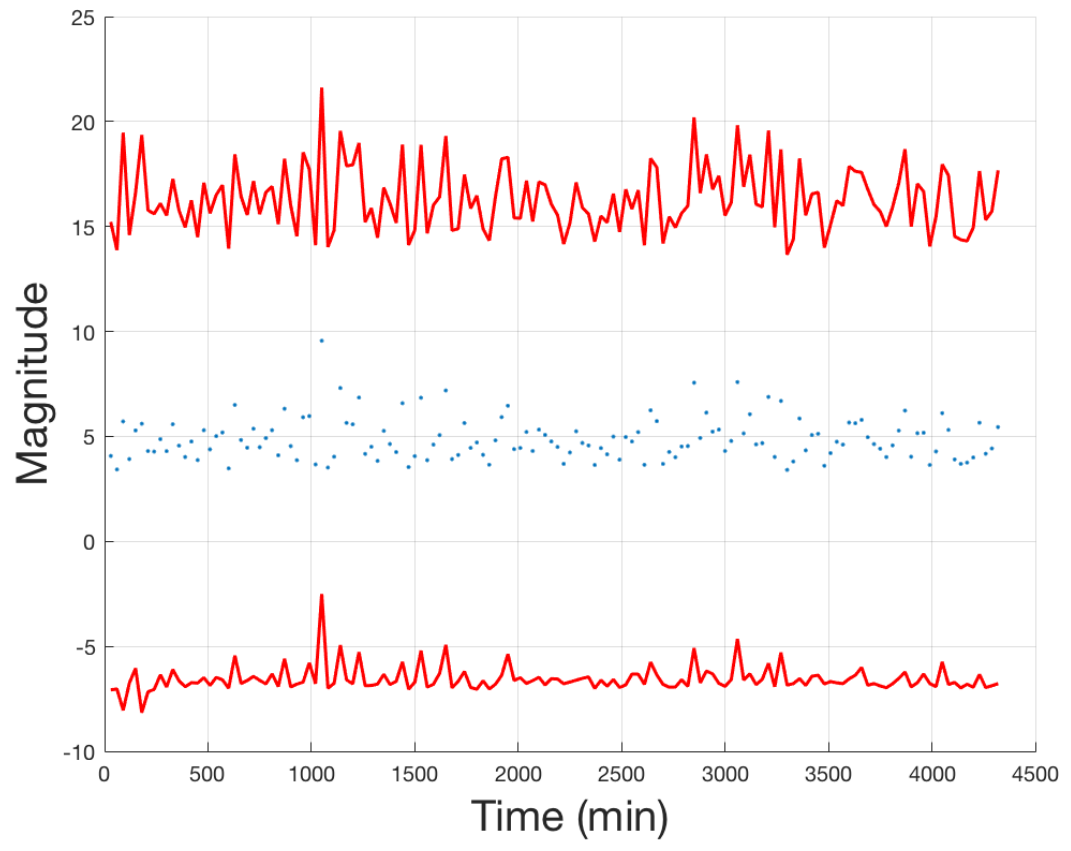

Figure A.7: Satellite 28868 apparent magnitude estimate with $3 \sigma$ error bounds.

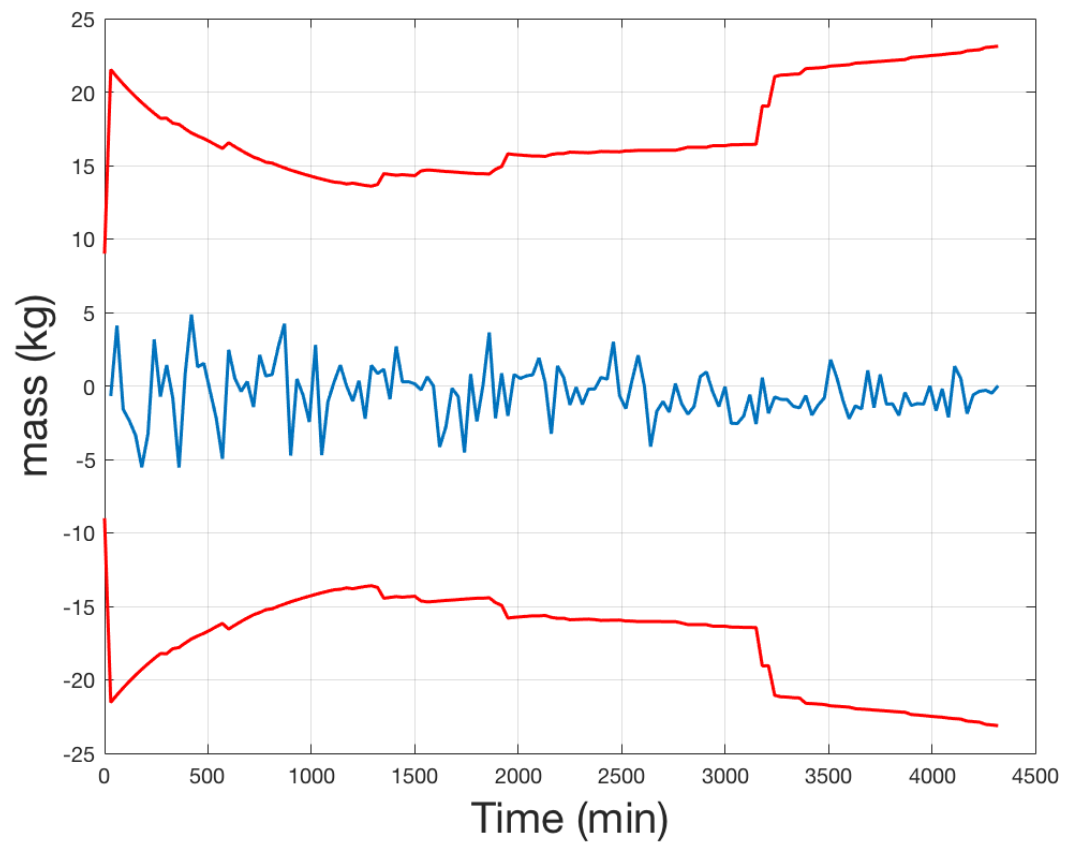

Figure A.8: Satellite 28868 mass estimate with $3 \sigma$ error bounds. 

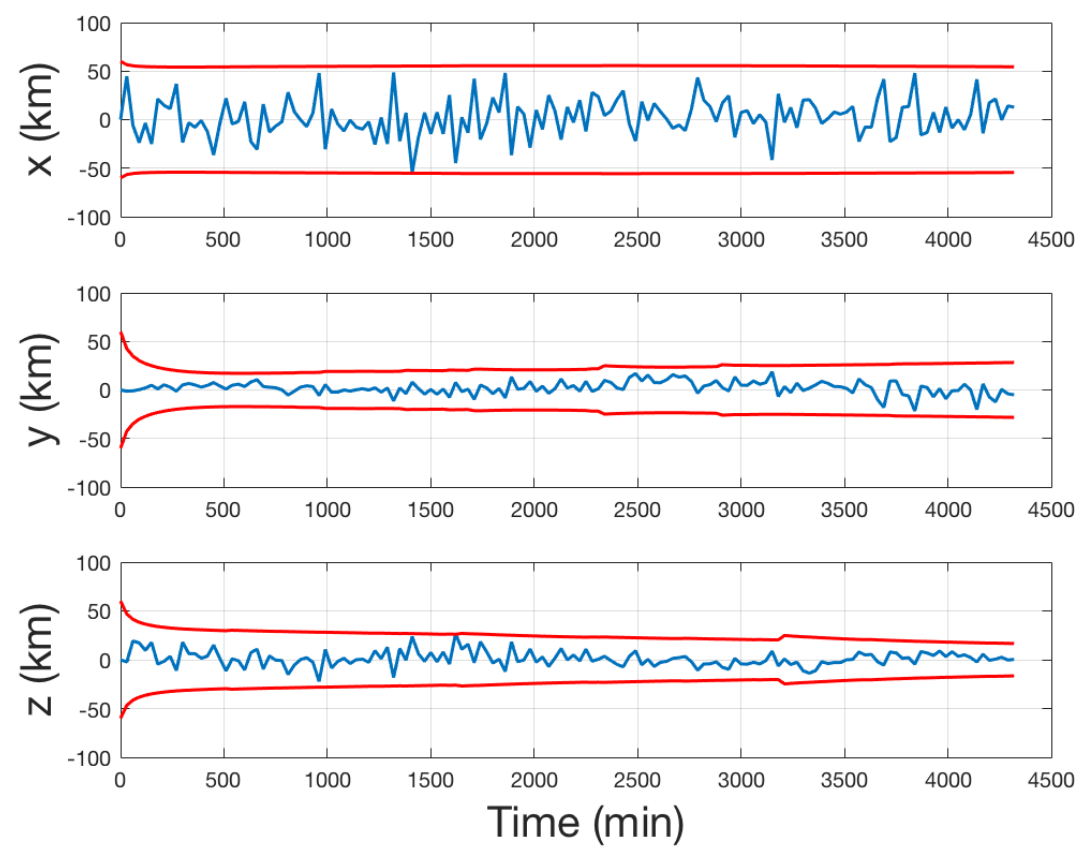

Figure A.9: Satellite 26464 position estimate with $3 \sigma$ error bounds.
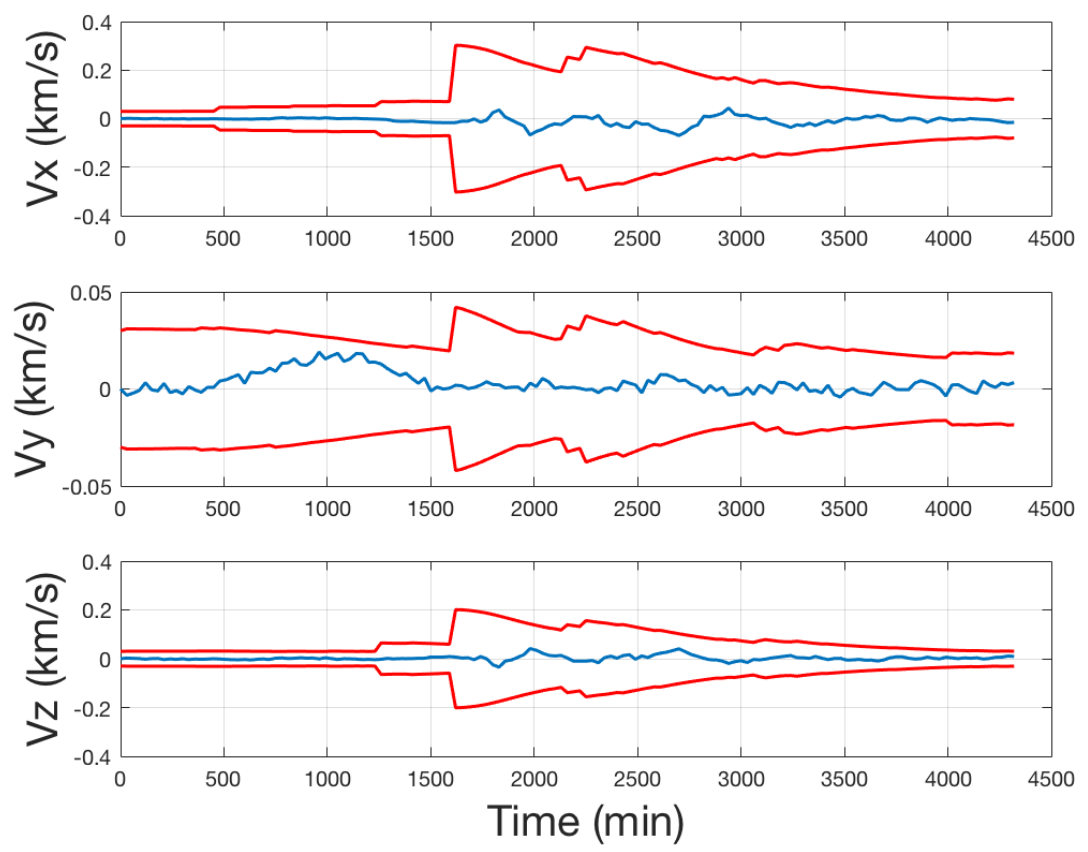

Figure A.10: Satellite 26464 velocity estimate with $3 \sigma$ error bounds. 


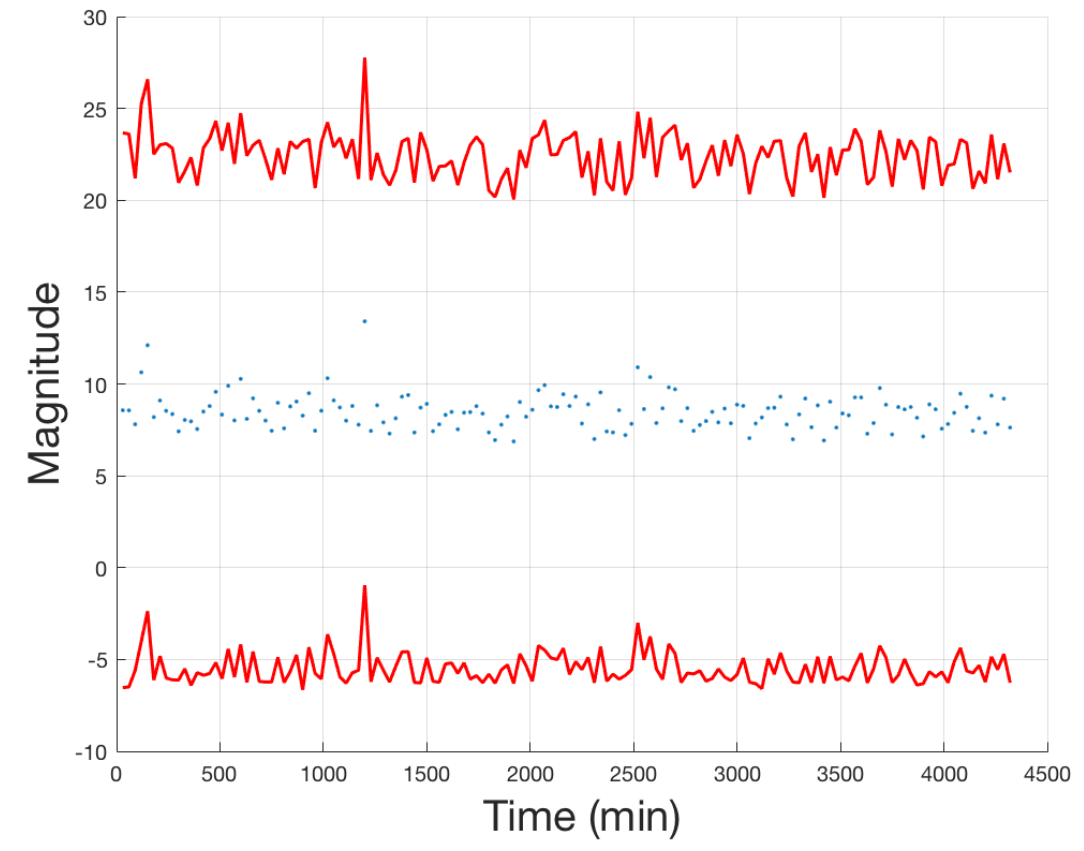

Figure A.11: Satellite 26464 apparent magnitude estimate with $3 \sigma$ error bounds.

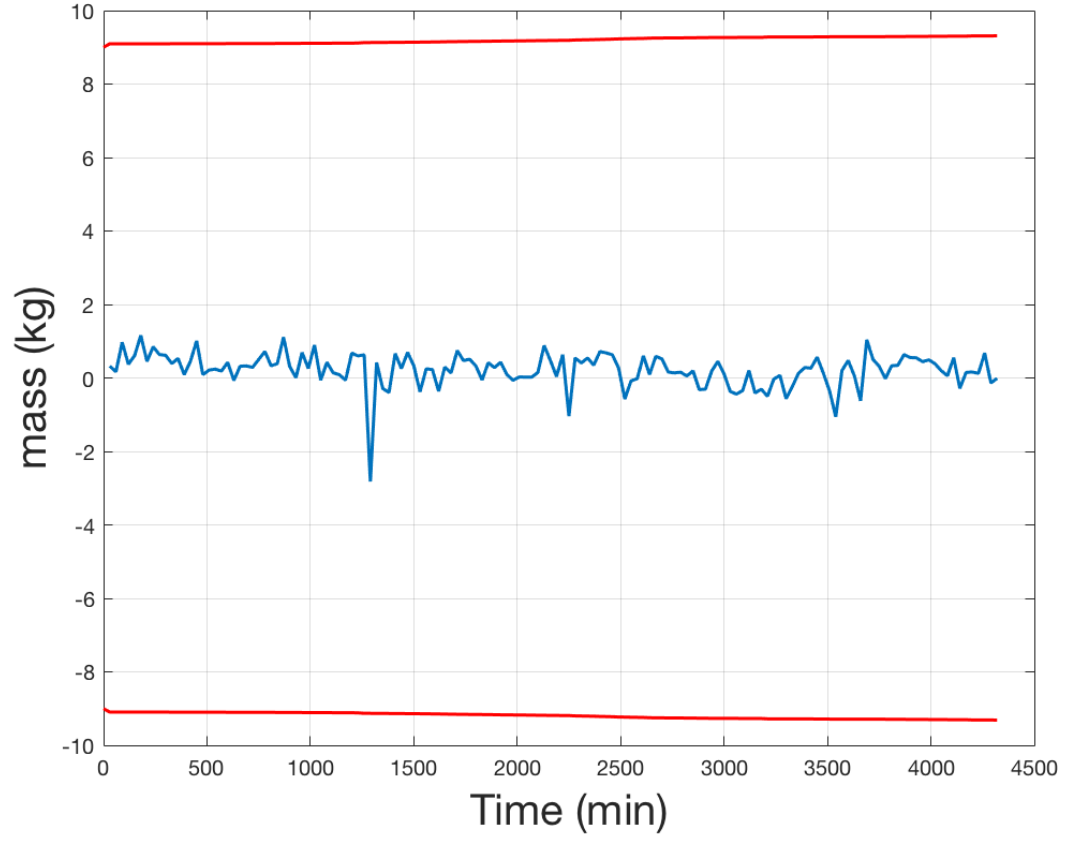

Figure A.12: Satellite 26464 mass estimate with $3 \sigma$ error bounds. 

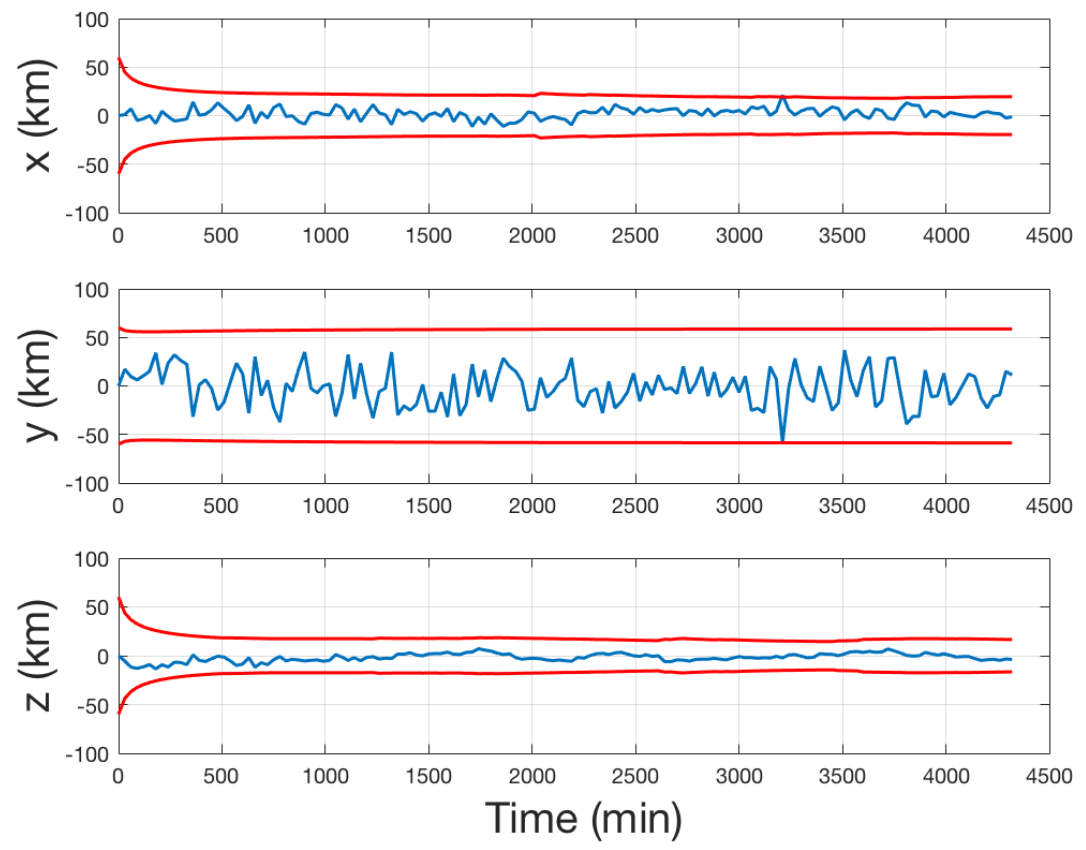

Figure A.13: Satellite 26483 position estimate with $3 \sigma$ error bounds.
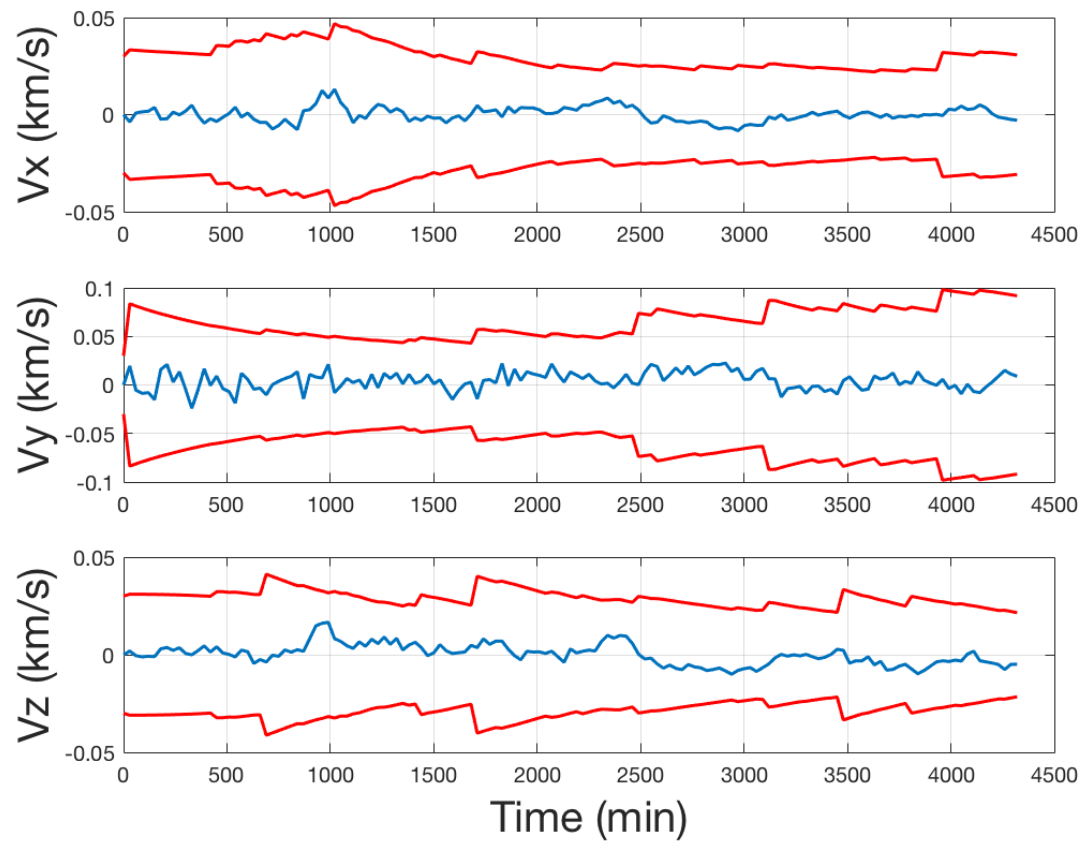

Figure A.14: Satellite 26483 velocity estimate with $3 \sigma$ error bounds. 


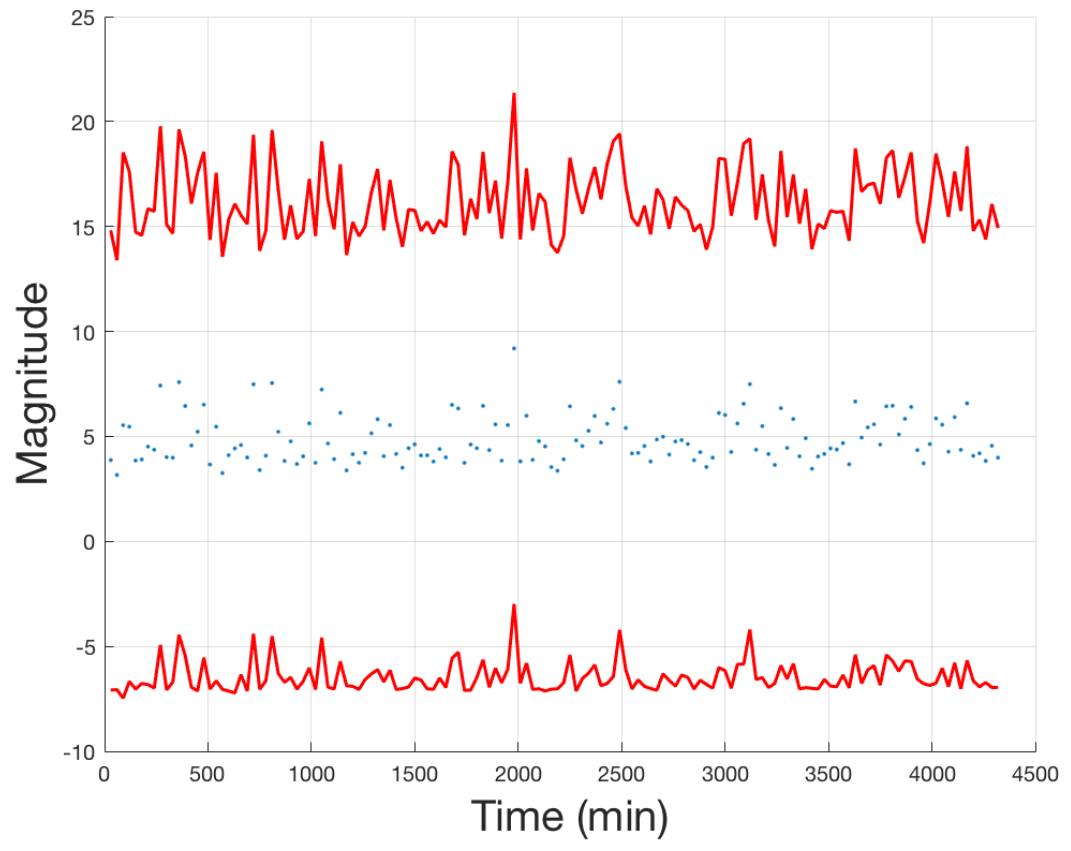

Figure A.15: Satellite 26483 apparent magnitude estimate with $3 \sigma$ error bounds.

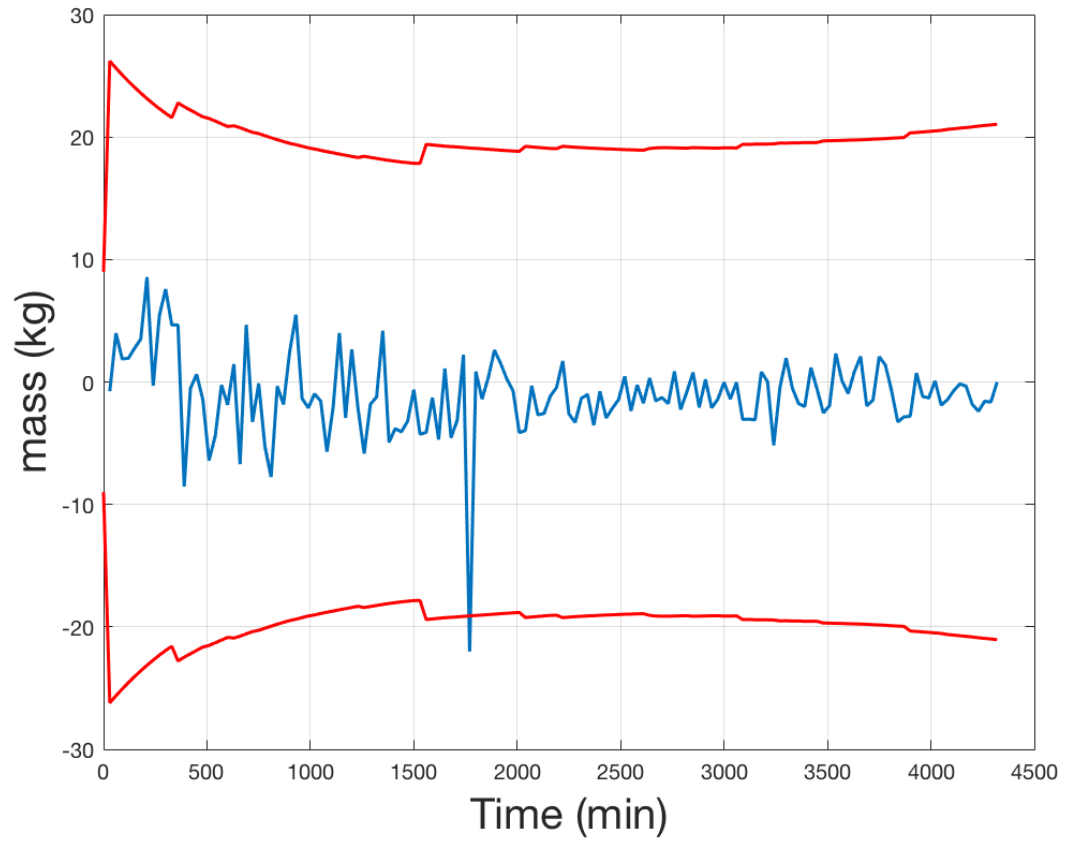

Figure A.16: Satellite 26483 mass estimate with $3 \sigma$ error bounds. 

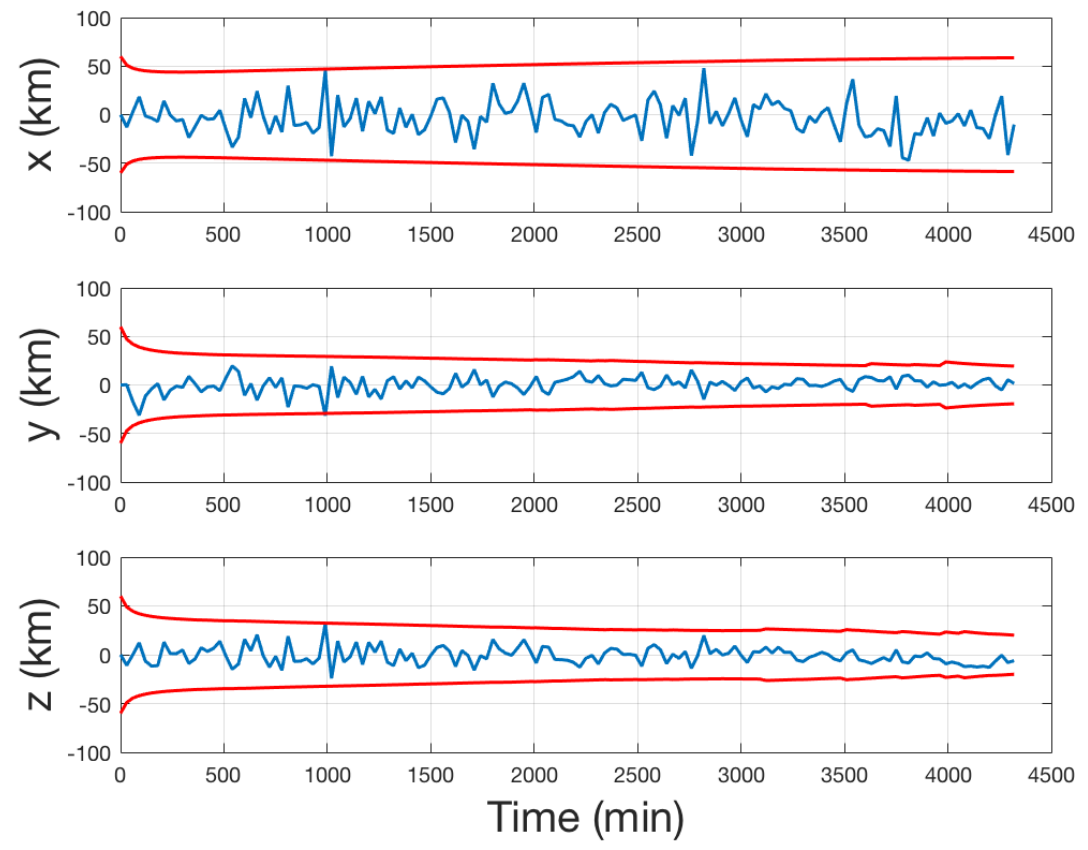

Figure A.17: Satellite 26690 position estimate with $3 \sigma$ error bounds.
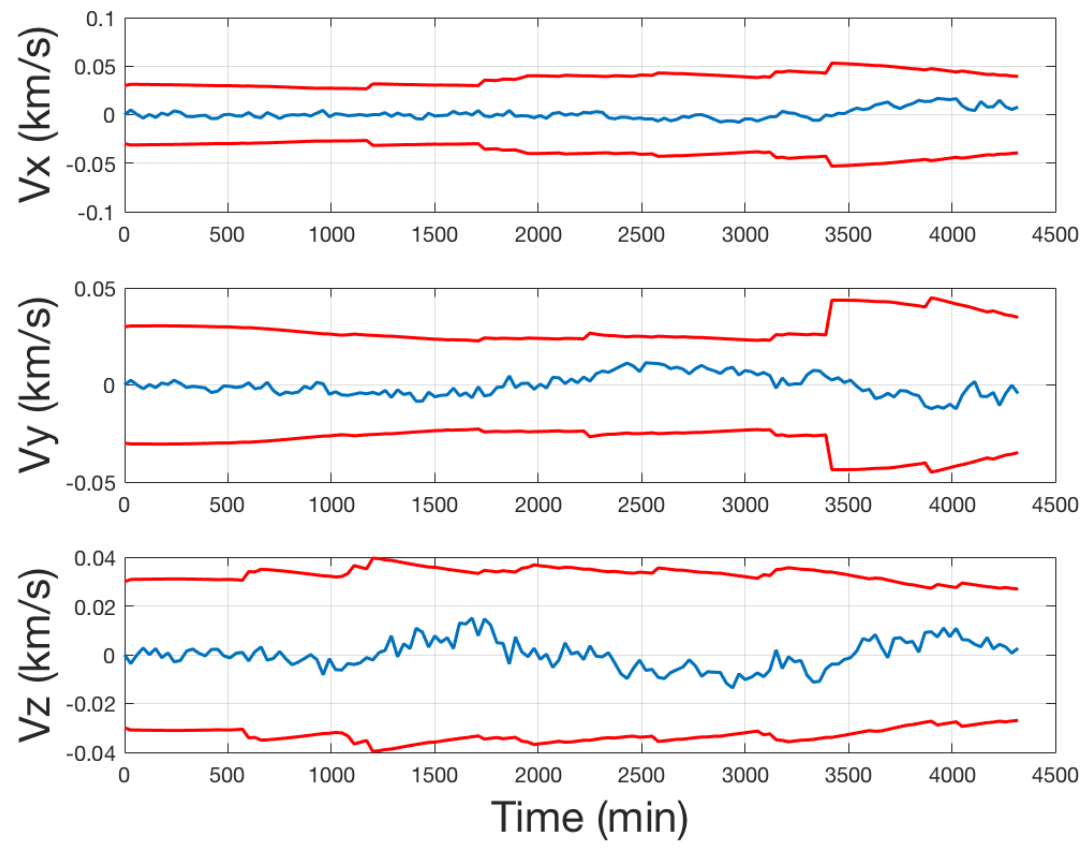

Figure A.18: Satellite 26690 velocity estimate with $3 \sigma$ error bounds. 


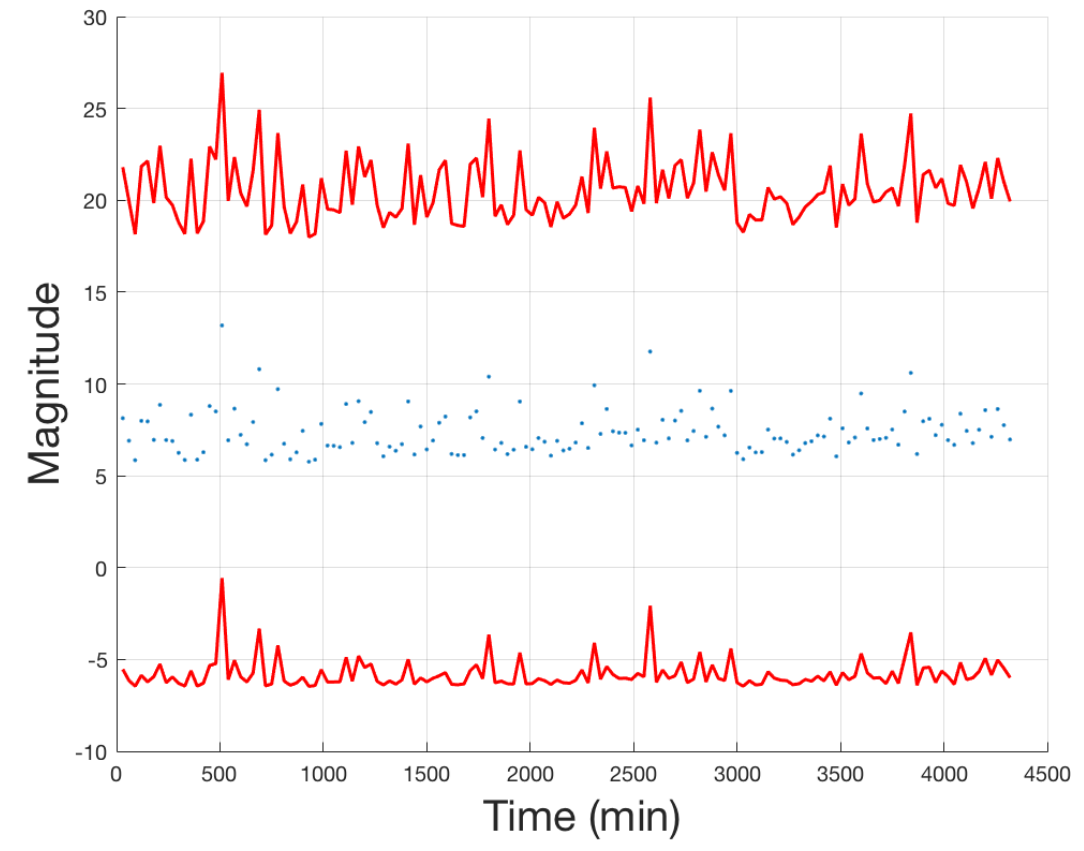

Figure A.19: Satellite 26690 apparent magnitude estimate with $3 \sigma$ error bounds.

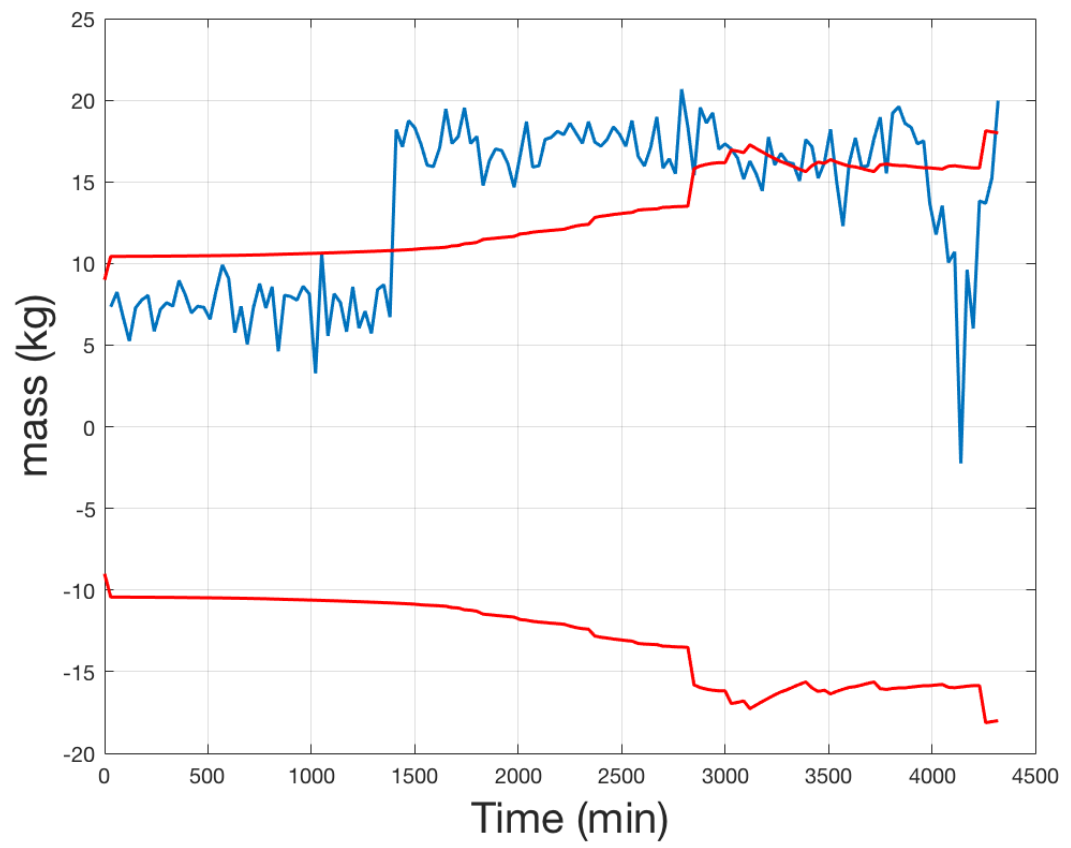

Figure A.20: Satellite 26690 mass estimate with $3 \sigma$ error bounds. 

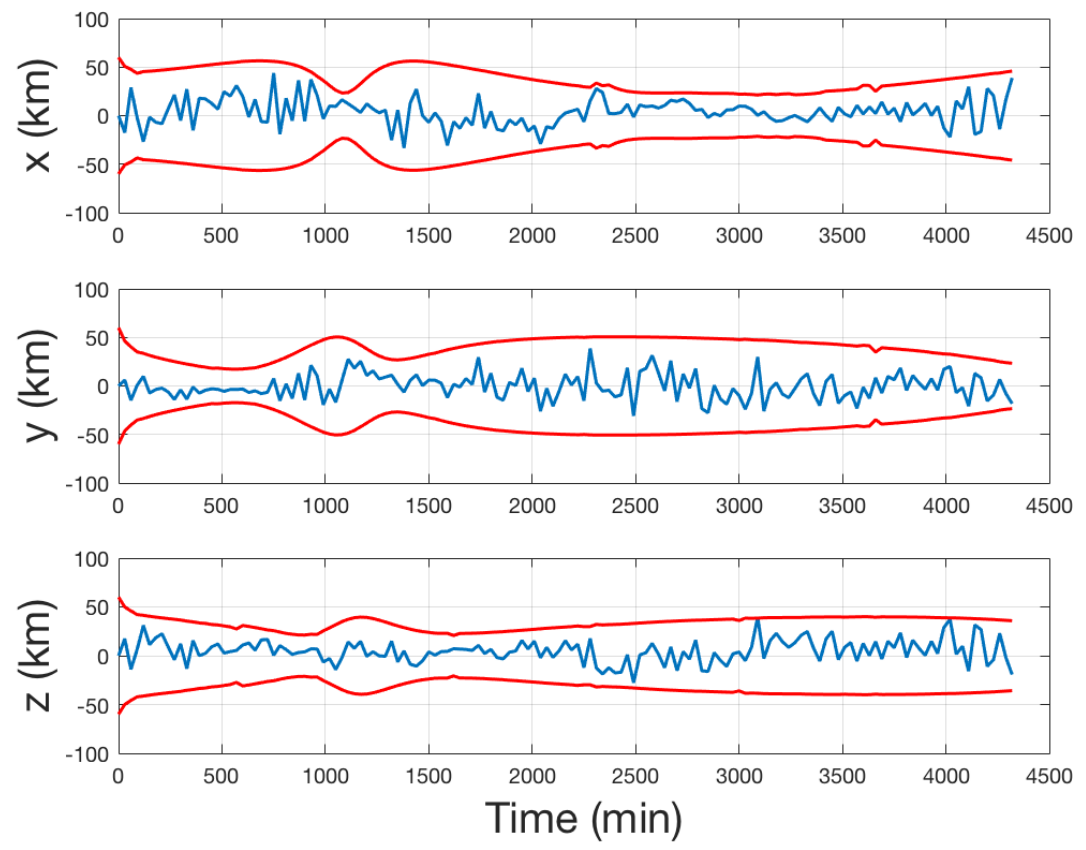

Figure A.21: Satellite 28874 position estimate with $3 \sigma$ error bounds.
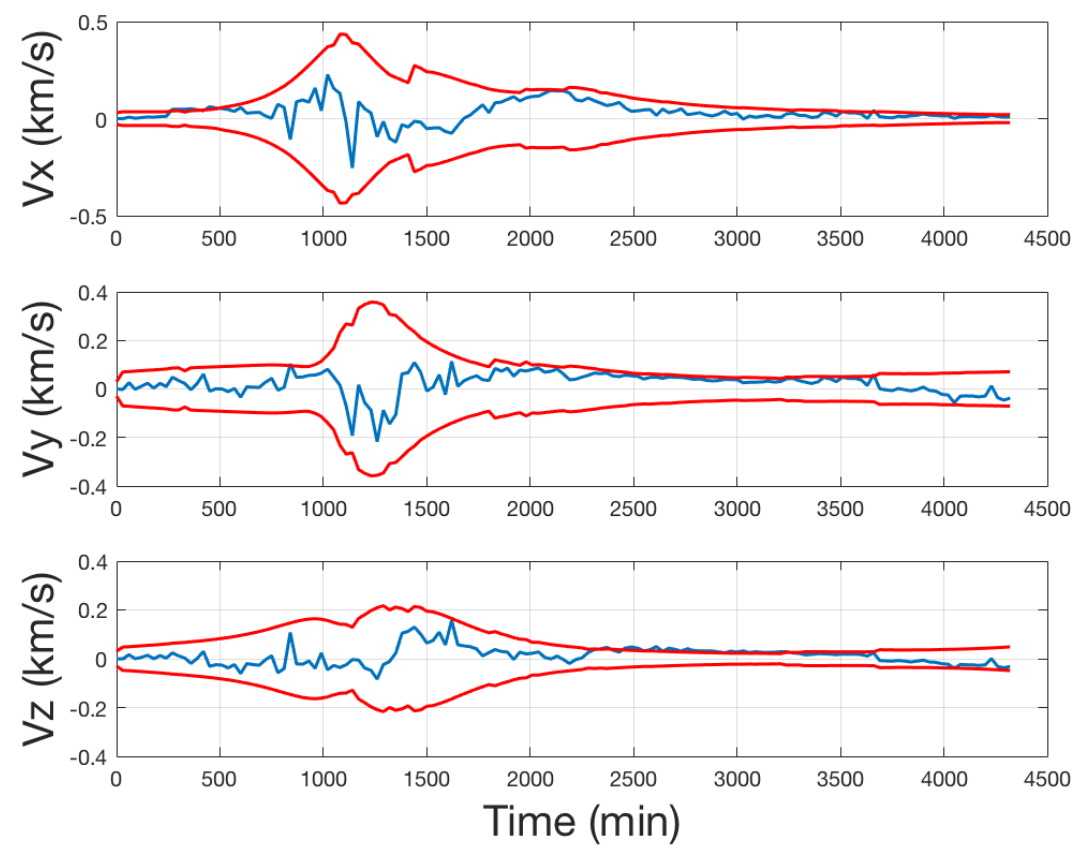

Figure A.22: Satellite 28874 velocity estimate with $3 \sigma$ error bounds. 


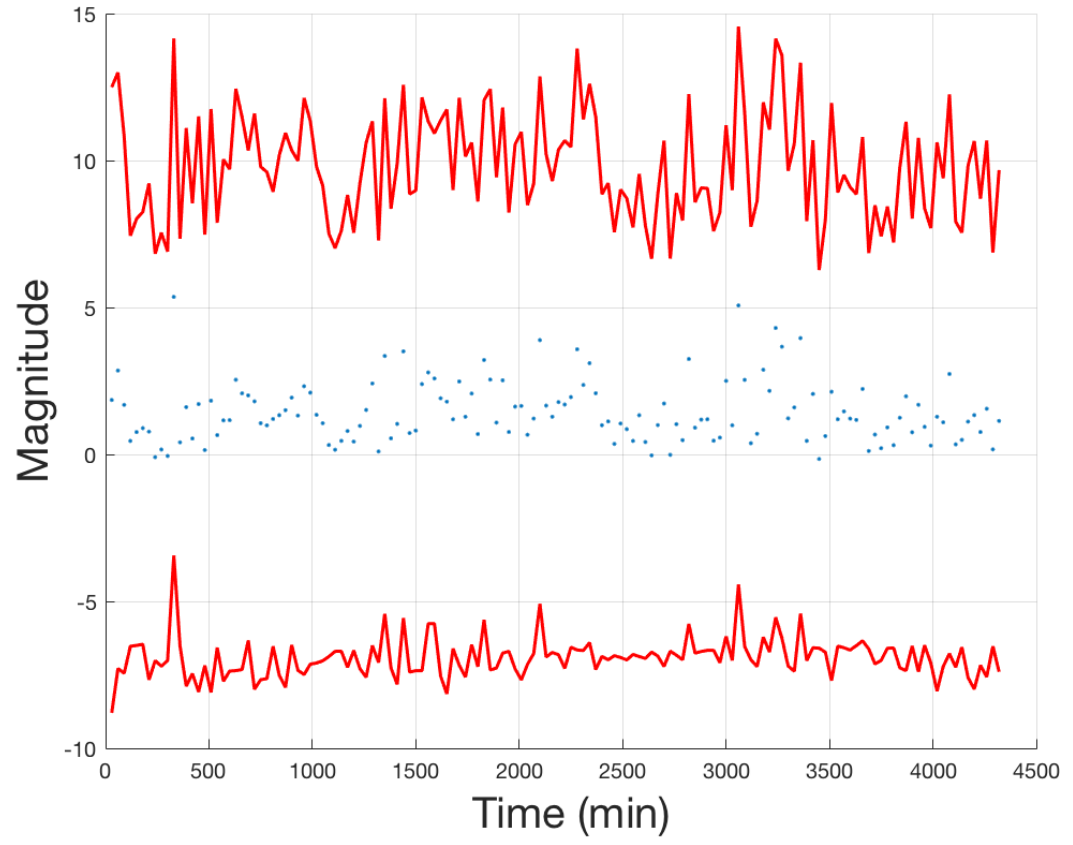

Figure A.23: Satellite 28874 apparent magnitude estimate with $3 \sigma$ error bounds.

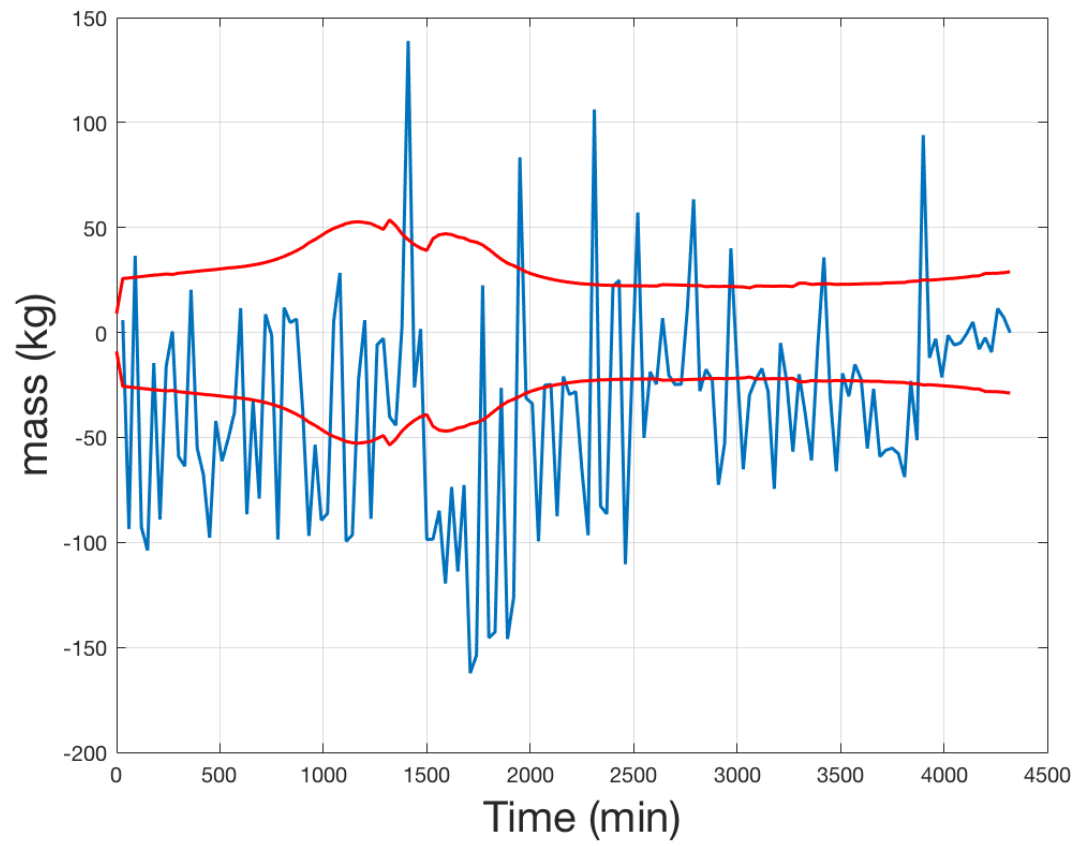

Figure A.24: Satellite 28874 mass estimate with $3 \sigma$ error bounds. 